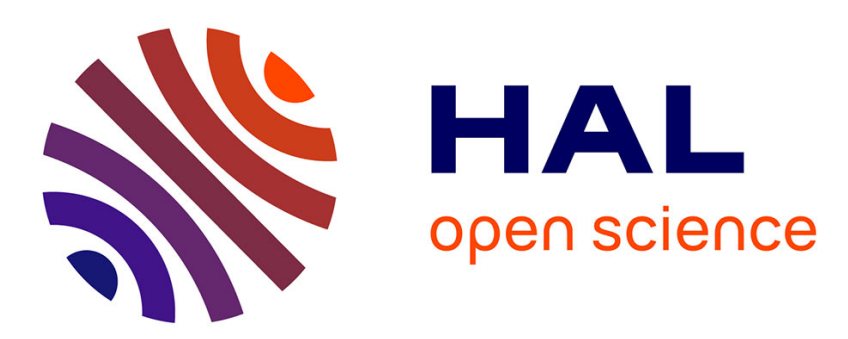

\title{
Stiffness and Strength Optimization of the Anisotropy Distribution for Laminated Structures
}

\author{
Anita Catapano, Boris Desmorat, Paolo Vannucci
}

\section{To cite this version:}

Anita Catapano, Boris Desmorat, Paolo Vannucci. Stiffness and Strength Optimization of the Anisotropy Distribution for Laminated Structures. Journal of Optimization Theory and Applications, 2015, pp.DOI 10.1007/s10957-014-0693-5. 10.1007/s10957-014-0693-5 . hal-01107512

\section{HAL Id: hal-01107512 \\ https://hal.science/hal-01107512}

Submitted on 21 Jan 2015

HAL is a multi-disciplinary open access archive for the deposit and dissemination of scientific research documents, whether they are published or not. The documents may come from teaching and research institutions in France or abroad, or from public or private research centers.
L'archive ouverte pluridisciplinaire HAL, est destinée au dépôt et à la diffusion de documents scientifiques de niveau recherche, publiés ou non, émanant des établissements d'enseignement et de recherche français ou étrangers, des laboratoires publics ou privés. 


\title{
Stiffness and Strength Optimization of the Anisotropy Distribution for Laminated Structures
}

\author{
Anita Catapano · Boris Desmorat · Paolo Vannucci
}

Received: date / Accepted: date

\begin{abstract}
In this paper, we deal with the problem of optimizing the anisotropy distribution of a laminated structure in order to maximize, simultaneously, its stiffness and strength. For these two objectives, two functionals are considered: the compliance as a measure of the plate stiffness and a laminate-level failure index as a measure of the strength. To solve this optimization problem we used a two-step hierarchical strategy: in the first step the aim is to find the best distribution of the stiffness and strength anisotropic tensors of an equivalent homogenized plate, while in the second one the objective is to find at least one laminate layup satisfying the optimal properties obtained as result of the first step. The polar formalism has been used to represent both the stiffness and strength tensors and allowed us to find analytical solutions to the local minimizations of the two functionals. A test case is finally presented to show the effectiveness of the proposed strategy.
\end{abstract}

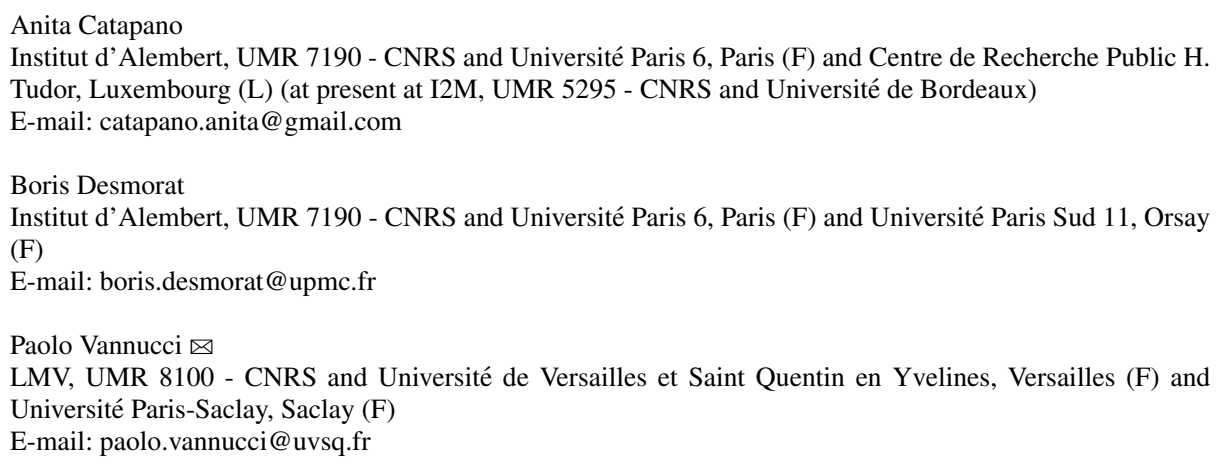


Keywords Anisotropy · Polar method · Stiffness and strength optimization

Mathematics Subject Classification (2000) (74E10) Anisotropy · (74B05) Classical linear elasticity $\cdot(74 \mathrm{P} 05)$ Compliance or weight optimization $\cdot(74 \mathrm{P} 10)$ Optimization of other properties

\section{Introduction}

Laminates are structures obtained by the superposition of several layers bonded together; to obtain light, robust and stiff structures, the layers are normally reinforced by straight fibers. Because of the orientation of the fibers, such layers, and subsequently also the laminates they compose, are anisotropic: all the physical properties depend upon the direction.

Modern technologies allow for the fabrication of a new kind of laminates, where the fibers reinforcing each layer are placed along given arbitrary curves. This opportunity opens a new optimization problem: to find, point-wise and layer by layer, the best tracing for the fibers, with the aim of minimizing the value of a given functional. All the mechanical properties, that are constant throughout the whole structure for metallic materials and also for laminates with straight fibers, are now functions of the position: they become scalar, vector or tensor fields defined over the structure. A general result is that the elastic behavior of a laminate is completely determined by three fourth rank tensors in $\mathbb{R}^{2}$; in some mechanical models, this is the same also for the strength behavior. As a consequence, mathematically speaking optimizing the stiffness or strength of Locally Dependent Laminates (abridged from now on in LDL) means to optimize the distribution of some anisotropic tensor fields.

While the first works on the optimization of laminates go back to the 70s [1-4], papers on LDL are rather recent [5-7]. A rather wide state of the art can be found in [4]. Following an old approach first introduced by Miki [8,9], and used in almost the totality of the works on the matter, we can subdivide each problem into two steps: in the first one, that we will call the structural problem, the aim is to find the best distribution of the anisotropic tensors describing the property to be optimized. In the second one, that we will call the lay-up problem, the objective is to find a sequence of orientation fields giving a laminate with the optimal properties obtained as result of the first step. Also this second step can be traduced 
into an optimization problem, namely in a problem of minimal distance, between the (actual) properties of the laminate and those (optimal) that are solution of the first step.

While in all of the previously cited works the objective of the research was the maximization of the stiffness, we consider here a problem where the objective is to find, for a given material and number of layers, the laminate which is at the same time the stiffest and strongest one.

It is important to remark that, contrarily to what happens in several cases with homogeneous, e.g. metallic, structures, the stiffest laminate is not necessarily the strongest one: the solutions can be different for the two cases. This happens because elastic and strength moduli are independent for a laminate. Hence, to look for the stiffest laminate is not necessarily concurrent with looking for the strongest one: the problem cannot be seen as a bi-objective one, but more precisely as a sort of a new problem, that could be stated alternatively in one of the two following ways: find, for the distribution of the elastic properties giving the stiffest laminate, the distribution of the strength properties giving the strongest structure or, dually, find, for the distribution of the strength properties giving the strongest laminate, the distribution of the elastic properties giving the stiffest structure. In the first case, stiffness is the leading property, and strength the secondary one, in the second problem it is exactly the contrary.

We will consider in this paper the above two problems; each one will be formulated as a two-step problem, the above mentioned structural and lay-up steps, the first one being in turn subdivided into two sub-problems, for the leading and secondary property. The first and second step are linked together not only by the optimal distribution, solution of the first step, but also by a set of constraints to be satisfied in the first step in order to obtain a mechanical solution that can be really realized by a laminate. These constraints are known as geometrical bounds, [10]. We discuss also the numerical procedure for the resolution of the two steps; the first one, is solved by an alternate directions algorithm, which is substantially derived from an algorithm proposed by Allaire and Khon [11, 12], while for the second one a genetic algorithm is used [13]. 


\section{Simultaneous Stiffness and Strength Optimization}

As previously said, we consider in this paper the simultaneous optimization of stiffness and strength of a LDL. The goal is to determine first the optimal value of the anisotropy parameters of stiffness and strength at each point in the structure domain, then the direction of the fibers, pointwise and layer by layer. Some precision must be given and assumptions made to have a well posed optimization problem; they are specified hereafter.

About the mechanical problem, we consider a laminate with geometry, boundary conditions and applied loads known and fixed. In particular we consider a laminate having the total thickness $h$ fixed and the same everywhere; this assumption constitutes the iso-perimetric constraint, without it the problem would be meaningless: the stiffest and strongest structure would be simply the one having an infinite thickness. In addition,

- the stacking sequence is composed by identical plies, orthotropic and linearly elastic up to their ultimate strength. Only the orientation of the fibers can vary;

- the laminate must be designed to be orthotropic and extension-bending uncoupled everywhere;

- the kinematic model of Kirchhoff is taken to describe the elastic response of the structure, [14];

- the limit state is modeled only by the Tsai-Hill failure criterion, [15, 16];

- the material parameters describing stiffness are independent from those describing strength. Mathematically this assumption allows for completely splitting stiffness and strength, except for the direction of orthotropy;

- the strength properties can be condensed in the components of an elasticity-like fourth order plane tensor, an assumption always tacitly done, [16];

- the polar formalism is used to describe the anisotropy fields, [17, 18].

As said in Sec. 1, a two-step approach is used; to be more specific:

- Step 1 - the structural problem: the laminated structure is modeled as a single-layer homogeneous structure; the anisotropy fields are optimized, leading to the optimal local stiffness and strength properties of the structure; 
- Step 2 - the lay-up problem: a stacking sequence (i.e. a set of layer orientations) is looked for at each point of the structure in order to obtain a LDL matching everywhere the optimal values of the polar parameters obtained at step 1; the design variables are the orientations of the $n$ layers composing the laminate.

The first step is in turn decomposed into two linked sub-problems, one for the leading property to be optimized, the other one for the secondary property. The only link between the two sub-problems composing the first step is the conservation of the orthotropy direction. This is a main point of the procedure, motivated by self evident physical reasons: the directions of the orthotropy axes for stiffness and strength must coincide everywhere.

Some other points concerning the procedure described in the next sections must be outlined since now:

- in the polar formalism, the mechanical properties are described through tensor invariants and orientation angles; this allows on one side for reducing the number of design variables and, on the other side, for obtaining some analytical solutions, speeding up the numerical resolution procedure;

- we will introduce a homogenized failure index as objective function for strength. This is a local functional giving a measure of the mechanical state of stress in a point;

- we use the compliance as objective function for stiffness; it is a global functional measuring the flexibility of the structure, so its minimization corresponds to the maximization of an averaged measure of the stiffness;

- the alternate directions optimization algorithm used for solving simultaneously the two sub-problems of the first step, alternates local and global minimizations; thus, it is capable of taking into account for the local character of the strength criterion;

- the correspondence between the elastic behavior of a laminate and the stacks is not bijective, hence, a lot of different stacks give rise to the same mechanical properties. This redundancy is a fundamental point that renders possible the existence of laminates satisfying the optimal requirements at the lay-up design step.

In this paper, we will focus uniquely on the extension behavior of the structure. The transpose of the procedure to the bending behavior is almost straightforward, but considering at 
the same time the two behaviors is today not yet possible: the determination of the feasible domain for the whole set of polar invariants of bending and extension is still a mathematical open problem.

\section{Polar Parametrization for Laminates}

Polar Formalism. The polar method, introduced by Verchery [17] in 1979, is a tensor representation based upon invariants; for a deeper presentation, see $[18,19]$. In the following, tensors axe expressed in the frame $1-2$ rotated counter clockwise through an angle $\theta$ with respect to the global frame $x-y$.

For any second order symmetrical stress tensor $\sigma$, the polar representation introduces the 2 polar moduli $T$ and $R$, which are invariants, and the angle $\Phi$ which gives the direction of the biggest principal stress in the frame 1-2 :

$$
\left\{\begin{array}{l}
\sigma_{11}=T+R \cos 2(\Phi-\theta) \\
\sigma_{22}=T-R \cos 2(\Phi-\theta) \\
\sigma_{12}=R \sin 2(\Phi-\theta)
\end{array}\right.
$$

For any orthotropic $4^{\text {th }}$ order stiffness tensor $\mathbb{Q}$, the polar representation introduces five invariants: four polar moduli $T_{0}, T_{1}, R_{0}, R_{1}$ and the orthotropy shape $K \in\{0,1\}$. An angle $\Phi_{1}$ is arbitrarily chosen to fix the principal orthotropy direction in the frame $1-2$ :

$$
\left\{\begin{array}{l}
Q_{1111}=T_{0}+2 T_{1}+(-1)^{K} R_{0} \cos 4\left(\Phi_{1}-\theta\right)+4 R_{1} \cos 2\left(\Phi_{1}-\theta\right) \\
Q_{1112}=\quad+(-1)^{K} R_{0} \sin 4\left(\Phi_{1}-\theta\right)+2 R_{1} \sin 2\left(\Phi_{1}-\theta\right) \\
Q_{1122}=-T_{0}+2 T_{1}-(-1)^{K} R_{0} \cos 4\left(\Phi_{1}-\theta\right), \\
Q_{1212}=T_{0} \quad-(-1)^{K} R_{0} \cos 4\left(\Phi_{1}-\theta\right) \\
Q_{2212}=\quad-(-1)^{K} R_{0} \sin 4\left(\Phi_{1}-\theta\right)+2 R_{1} \sin 2\left(\Phi_{1}-\theta\right), \\
Q_{2222}=T_{0}+2 T_{1}+(-1)^{K} R_{0} \cos 4\left(\Phi_{1}-\theta\right)-4 R_{1} \cos 2\left(\Phi_{1}-\theta\right)
\end{array}\right.
$$

The polar moduli are all positive or non-negative:

$$
T_{0}>0, \quad T_{1}>0, \quad R_{0} \geq 0, \quad R_{1} \geq 0 .
$$


The positive definiteness of the orthotropic stiffness tensor gives the bounds

$$
\left\{\begin{array}{l}
T_{0}-R_{0}>0 \\
T_{1}\left(T_{0}+(-1)^{K} R_{0}\right)-2 R_{1}^{2}>0
\end{array}\right.
$$

Application to laminates. Let us consider a laminated plate made by $n$ identical plies, i.e. with the same thickness and made of the same material. The material behaviour of the elementary ply is linear elastic orthotropic and the corresponding polar parameters are noted $T_{0}, T_{1}, R_{0}, R_{1}, K$ and $\Phi_{1}$. Each $k^{\text {th }}$ ply is characterized by its position $z_{k}$ with respect to the mid-plane, its orientation $\delta_{k}$ with respect to the $x$ axis and its elastic properties defined by the in-plane stiffness tensor $\mathbb{Q}\left(\delta_{k}\right)$. The constitutive law of the plate can be written as [16]:

$$
\begin{aligned}
& \mathbf{N}=h \mathbb{A} \varepsilon_{0}+\frac{h^{2}}{2} \mathbb{B} \chi \\
& \mathbf{M}=\frac{h^{2}}{2} \mathbb{B} \varepsilon_{0}+\frac{h^{3}}{12} \mathbb{D} \chi .
\end{aligned}
$$

with $\varepsilon_{0}$ and $\chi$ the in-plane strain and curvature tensors of the mid-plane of the plate, while $\mathbf{N}$ and $\mathbf{M}$ are the tensors of in-plane forces and bending moments. $\mathbb{A}, \mathbb{B}$ and $\mathbb{D}$ are the extension, membrane-bending coupling and bending stiffness tensors of the homogenized laminated plate, defined by (with $m=1,2,3$ and $c=1,2,12$ for $\mathbb{A}, \mathbb{B}$ and $\mathbb{D}$ respectively):

$$
\mathbb{A}, \mathbb{B}, \mathbb{D}=\frac{c}{m h^{m}} \sum_{k=1}^{n} \mathbb{Q}\left(\delta_{k}\right)\left(z_{k}^{m}-z_{k-1}^{m}\right) .
$$

The laminate behaviour is supposed to be uncoupled and orthotropic in membrane and in bending. The polar parameters $\left(\bar{T}_{0}, \bar{T}_{1}, \bar{R}_{0}, \bar{R}_{1}, \bar{K}, \bar{\Phi}_{1}\right)$ of $\mathbb{A}$ can be given in terms of those of the elementary ply $\left(T_{0}, T_{1}, R_{0}, R_{1}, K\right)$, and with the choice $\Phi_{1}=0$ :

$$
\left\{\begin{array}{l}
\bar{T}_{0}=T_{0} \\
\bar{T}_{1}=T_{1} \\
(-1)^{\bar{K}} \bar{R}_{0} e^{4 i \bar{\Phi}_{1}}=\frac{1}{n}(-1)^{K} R_{0} \sum_{k=1}^{n} e^{4 i \delta_{k}} \\
\bar{R}_{1} e^{2 i \bar{\Phi}_{1}}=\frac{1}{n} R_{1} \sum_{k=1}^{n} e^{2 i \delta_{k}}
\end{array}\right.
$$


Eq. (7) shows that the isotropic polar parameters of the laminate are equal to the isotropic polar parameters of the elementary ply.

The polar formalism allows to write explicitly the conditions on the polar parameters, called geometric bounds [10], in order to ensure the existence of a stacking sequence which satisfies equation (7):

$$
\left\{\begin{array}{l}
2\left(\frac{\bar{R}_{1}}{R_{1}}\right)^{2}-1 \leq \frac{(-1)^{\bar{K}} \bar{R}_{0}}{(-1)^{K} R_{0}}, \\
\left|(-1)^{\bar{K}} \bar{R}_{0}\right| \leq R_{0}, \\
\bar{R}_{1} \geq 0
\end{array}\right.
$$

The geometric bounds (8) define a subset of the domain defined by (4), when written for $\mathbb{A}$, [10]. Conditions similar to (7) and (8) hold also for $\mathbb{D}$.

\section{Evaluation of the Laminate Strength Using a Homogenized Criterion}

In [20], some polynomial failure criteria are formulated using invariants defined by the polar method, and the optimal orientation that maximizes the strength of a linearly elastic orthotropic sheet is determined. With the aim of extending this analytical approach to the strength optimization of laminated plates, we define in this section a homogenized failure criterion used as a measure of the strength for the laminate, and give its link with the failure index of each individual ply that composes the laminate. The failure criterion of Tsai-Hill is taken here as strength measure, but other criteria could be chosen as well without essentially changing the procedure.

The stress-based polynomial failure criterion of Tsai-Hill $[15,16]$, in the $2 \mathrm{D}$ space for a generic orthotropic ply can be put in the following form [20]:

$$
F_{\text {Hill }}^{\text {Ply }} \leq 1 \quad \text { with } \quad F_{H i l l}^{P l y}=\sigma \cdot \mathbb{F} \sigma
$$

in which $F_{H i l l}^{P l y}$ is the failure index of the ply considered and $\mathbb{F}$ is the fourth order elasticitylike weakness tensor. Introducing the Hooke's law $\sigma=\mathbb{Q} \varepsilon$, the previous equations reads:

$$
F_{H i l l}^{\text {Ply }} \leq 1 \quad \text { with } \quad F_{H i l l}^{P l y}=\varepsilon \cdot \mathbb{G} \varepsilon \quad \text { and } \quad \mathbb{G}=\mathbb{Q} \mathbb{F} \mathbb{Q}
$$


$\mathbb{G}$ is the strength tensor; it allows to express the stress-based failure criterion in terms of strains. Assumed to be orthotropic, it has the same symmetries of tensors $\mathbb{Q}$ and $\mathbb{F}$. Its polar parameters are denoted by the isotropic moduli $\Gamma_{0}$ and $\Gamma_{1}$, the anisotropic parameters $\Lambda_{0}$, $\Lambda_{1}, L$ and by the orthotropy direction $\Omega_{1}$. The orthotropy type of the strength tensor $\mathbb{G}$ is not directly linked the orthotropy type of $\mathbb{Q}$ or $\mathbb{F}$ (depending on the elementary ply, the principal orthotropy axis of the stiffness tensor $\mathbb{Q}$ and of the weakness tensor $\mathbb{F}$ may be the same or turned by $\pi / 2$ [21]). Therefore the principal orthotropy direction of $\mathbb{G}$ may be aligned or orthogonal to the principal orthotropy direction of the stiffness tensor $\mathbb{Q}$ :

$$
\Omega_{1}=\Phi_{1} \quad \text { or } \quad \Phi_{1}+\frac{\pi}{2}
$$

The homogenized failure criterion of the laminate is defined as:

$$
F_{\text {Hill }}^{\text {Lam }} \leq 1 \quad \text { with } \quad F_{\text {Hill }}^{\text {Lam }}=\frac{1}{h} \int_{-\frac{h}{2}}^{\frac{h}{2}} F_{\text {Hill }}^{\text {Ply }}(z) d z
$$

in which $F_{H i l l}^{\text {Lam }}$ is the failure index of the laminate.

The strain field for the laminate has the form $\varepsilon=\varepsilon_{0}+z \chi$. Since the in-plane strain tensor $\varepsilon_{0}$ and the curvature tensor $\chi$ do not depend upon $z$ we get:

$$
F_{\text {Hill }}^{\text {Lam }}=\left(\varepsilon_{0} \cdot \mathbb{G}^{A} \varepsilon_{0}+\chi \cdot \mathbb{G}^{D} \chi+2 \varepsilon_{0} \cdot \mathbb{G}^{B} \chi\right),
$$

with ( $m=1,2,3$ for $\mathbb{G}^{A}, \mathbb{G}^{B}$ and $\mathbb{G}^{D}$ respectively):

$$
\mathbb{G}^{A}, \mathbb{G}^{B}, \mathbb{G}^{D}=\frac{1}{m h} \sum_{k=1}^{n} \mathbb{G}\left(\delta_{k}\right)\left(z_{k}^{m}-z_{k-1}^{m}\right) .
$$

For laminates made with identical plies, uncoupling in stiffness is equivalent to uncoupling in strength: $\mathbb{B}=0 \Leftrightarrow \mathbb{G}^{B}=0$. Considering an uncoupled laminate (thus both in stiffness and strength) and only membrane loadings (i.e. $\chi=0)$, eq. (13) leads to:

$$
F_{\text {Hill }}^{\text {Lam }}=\varepsilon_{0} \cdot \mathbb{G}^{A} \varepsilon_{0}
$$


The polar parameters $\bar{\Gamma}_{0}, \bar{\Gamma}_{1}, \bar{\Lambda}_{0}, \bar{\Lambda}_{1}, \bar{L}$ and $\bar{\Omega}_{1}$ of $\mathbb{G}^{A}$ can be expressed in terms of the strength polar parameters of the elementary ply $\left(\Gamma_{0}, \Gamma_{1}, \Lambda_{0}, \Lambda_{1}, L\right)$, using eq. (11) and the choice $\Phi_{1}=0$ (see eq. (7)):

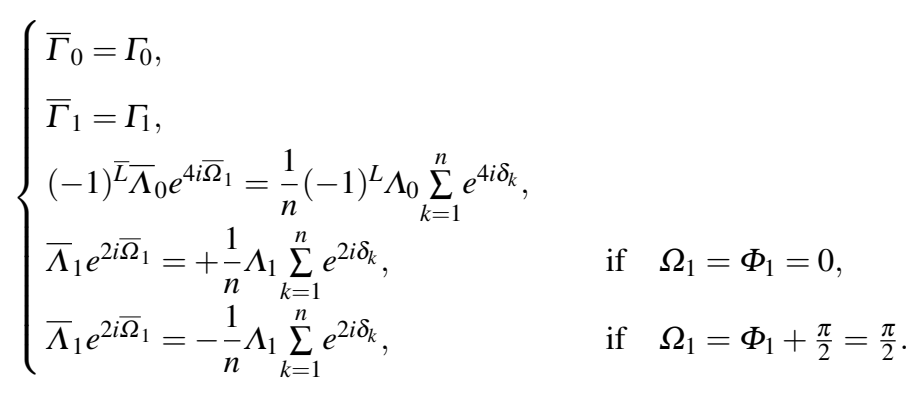

Eqs. (16) impose geometric bounds on the polar parameters of $\mathbb{G}^{A}$ similar to those already introduced for $\mathbb{A}$ in eq. (8):

$$
\left\{\begin{array}{l}
2\left(\frac{\bar{\Lambda}_{1}}{\Lambda_{1}}\right)^{2}-1 \leq \frac{(-1)^{\bar{L}} \bar{\Lambda}_{0}}{(-1)^{L} \Lambda_{0}}, \\
\left|(-1)^{\bar{L}} \bar{\Lambda}_{0}\right| \leq \Lambda_{0}, \\
\bar{\Lambda}_{1} \geq 0
\end{array}\right.
$$

For an uncoupled laminate subjected to a pure membrane loading, the strain field $\varepsilon$ in each constitutive layer is equal to the in-plane strain tensor $\varepsilon_{0}$. The polar parameters of the strain field $\varepsilon$ are denoted by $t, r$ and $\varphi$. In terms of polar parameters, the failure indexes of the laminate and of the $k^{\text {th }}$ constitutive layer are, respectively:

$$
\begin{aligned}
& F_{\text {Hill }}^{\text {Lam }}=4 r^{2} \Gamma_{0}+8 t^{2} \Gamma_{1}+4(-1)^{\bar{L}} \bar{\Lambda}_{0} r^{2} \cos 4\left(\bar{\Omega}_{1}-\varphi\right)+16 t r \bar{\Lambda}_{1} \cos 2\left(\bar{\Omega}_{1}-\varphi\right), \\
& F_{H i l l}^{P l y}=4 r^{2} \Gamma_{0}+8 t^{2} \Gamma_{1}+4(-1)^{L} \Lambda_{0} r^{2} \cos 4\left(\Omega_{1}^{(k)}-\varphi\right)+16 t r \Lambda_{1} \cos 2\left(\Omega_{1}^{(k)}-\varphi\right),
\end{aligned}
$$

in which $\Omega_{1}^{(k)}=\delta^{(k)}$ or $\delta^{(k)}+\frac{\pi}{2}$. For a given layup and strain, the failure index of the laminate is given. For each ply all the quantities are fixed, except for the principal orthotropy direction $\Omega_{1}^{(k)}$ of the strength tensor.

It is then possible to evaluate the orthotropy orientation $\Omega_{1}^{(k)}$ of the $k^{\text {th }}$ ply which maximizes the failure index of the ply $F_{H i l l}^{P l y}$. Such a case represents the worst situation that can be achieved in any ply of any possible layup (with identical plies). 
The problem of finding the orientation $\Omega_{1}$ maximizing, for a generic ply, the failure index, is formulated as follows:

$$
\max _{\Omega_{1}} F_{H i l l}^{P l y}\left(\Gamma_{0}, \Gamma_{1}, \Lambda_{0}, \Lambda_{1}, L, \Omega_{1}, t, r, \varphi\right)
$$

This problem comes down to finding the maximal value of the continuous periodic function $F_{H i l l}^{P l y}$ of the angle parameter $\Omega_{1}$. The study of the stationnary points is similar to the studies presented in [22, section 4] or in [20, section 5.1] where the minimum of respectively the complementary energy and the Hill-Tsai failure index expressed using the polar formalism was searched.

Two sets of solutions exist, depending on the value of $\mathrm{L}$ :

- for $L=0: \Omega_{1}^{o p t}=\operatorname{dir}\left(\max \left\{\left|\varepsilon_{I}\right|,\left|\varepsilon_{I I}\right|\right\}\right)$

- for $L=1$ :

- if $\frac{\Lambda_{1}|t|}{\Lambda_{0} r} \geq 1: \Omega_{1}^{o p t}=\operatorname{dir}\left(\max \left\{\left|\varepsilon_{I}\right|,\left|\varepsilon_{I I}\right|\right\}\right)$

- if $\frac{\Lambda_{1}|t|}{\Lambda_{0} r} \leq 1: \Omega_{1}^{o p t}=\operatorname{dir}\left(\max \left\{\left|\varepsilon_{I}\right|,\left|\varepsilon_{I I}\right|\right\}\right) \pm \arccos \left[\frac{\Lambda_{1}|t|}{\Lambda_{0} r}\right]$

In conclusion, we are able to evaluate a priori the maximum value of $F_{H i l l}^{P l y}$ at the end of the first step, the structural problem, i.e. when the lay-up is still unknown. If this value is lower than one, the search for a lay-up will be performed without any constraint related to the first-ply failure criterion. If not, it will be possible to impose a restriction on the orientations $\delta^{(k)}$ of the plies during the search for an optimal layup, see Sec. 7.

\section{First Step : Statement of the Structural Optimization Problem}

Two objective functions are considered. The plate stiffness is measured by the compliance, which is equal to twice the complementary energy:

$$
\text { Compliance }=\frac{1}{h} \int_{S_{p}} \mathbf{N} \cdot \mathbb{A}^{-1}\left(\bar{\Phi}_{1}, \bar{R}_{0 K}, \bar{R}_{1}\right) \mathbf{N} d S
$$

where $\bar{R}_{0 K}=(-1)^{\bar{K}} \bar{R}_{0}$. This objective function is a global functional, i.e. defined over the entire surface $S_{p}$ of the plate. 
The strength of the laminate is measured by the homogenized failure index $F_{H i l l}^{\mathrm{Lam}}$ introduced in Sec. 4:

$$
F_{\text {Hill }}^{\text {Lam }}=\varepsilon \cdot \mathbb{G}^{A}\left(\bar{\Omega}_{1}, \bar{\Lambda}_{0 L}, \bar{\Lambda}_{1}\right) \varepsilon
$$

where $\bar{\Lambda}_{0 L}=(-1)^{\bar{L}} \bar{\Lambda}_{0}$. This objective is a local functional, i.e. defined at each point of the plate.

The structural optimization parameters are the 6 polar parameters of the homogenized plate, i.e. $\left(\bar{\Phi}_{1}, \bar{R}_{0 K}, \bar{R}_{1}\right)$ for the stiffness tensor $\mathbb{A}$ and $\left(\bar{\Omega}_{1}, \bar{\Lambda}_{0 L}, \bar{\Lambda}_{1}\right)$ for $\mathbb{G}^{A}$, respectively. We assume, for the structural optimization step, that the stiffness anisotropic polar parameters, $\bar{R}_{0 K}$ and $\bar{R}_{1}$, are independent from the corresponding parameters for strength, $\bar{\Lambda}_{0 L}$ and $\bar{\Lambda}_{1}$ (see Sec. 2).

We consider the problem in which the compliance (20) and the laminate failure index (21) are to be minimized simultaneously. The optimization problem we are facing is then written as follows :

$$
\begin{gathered}
\left\{\begin{array} { l } 
{ \{ \overline { \Phi } _ { 1 } , \overline { R } _ { 0 K } , \overline { R } _ { 1 } \} } \\
{ \text { subject to } }
\end{array} \left\{\begin{array}{l}
2\left(\frac{\bar{R}_{1}}{R_{1}}\right)^{2}-1 \leq \frac{\bar{R}_{0 K}}{(-1)^{K} R_{0}}, \\
\left|\bar{R}_{0 K}\right| \leq R_{0}, \\
\bar{R}_{1} \geq 0 .
\end{array} \quad \forall x \in S_{p}\right.\right. \\
\forall x \in S_{p}\left\{\begin{array}{l}
\left\{\bar{\Omega}_{1}, \bar{\Lambda}_{0 L}, \bar{\Lambda}_{1}\right\} \\
\text { subject to } F_{\text {Hill }}^{\text {Lam }}
\end{array}\right. \\
\left\{\begin{array}{l}
2\left(\frac{\bar{\Lambda}_{1}}{\Lambda_{1}}\right)^{2}-1 \leq \frac{\bar{\Lambda}_{0 L}}{(-1)^{L} \Lambda_{0}}, \\
\left|\bar{\Lambda}_{0 L}\right| \leq \Lambda_{0}, \\
\bar{\Lambda}_{1} \geq 0 .
\end{array}\right.
\end{gathered}
$$

In order to solve the design problem (22a)-(22b), we use the assumption that stiffness and strength tensors share the same orthotropy direction, see eq. (11). In this way the number of independent design variables is reduced to five: $\bar{R}_{0 K}, \bar{R}_{1}, \bar{\Lambda}_{0 L}, \bar{\Lambda}_{1}$ plus the orthotropy orientation specified by $\bar{\Phi}_{1}$ or $\bar{\Omega}_{1}$, and two different approaches will be considered. The first approach will consider the stiffness as the leading objective, i.e. the stiffness orthotropy 
orientation is defined by solving the optimization problem (22a) and the problem (22b) is solved with a given strength orientation $\bar{\Omega}_{1} \in\left\{\bar{\Phi}_{1}, \bar{\Phi}_{1}+\frac{\pi}{2}\right\}$. The second approach will consider the strength as the leading objective, i.e. the strength orthotropy direction $\bar{\Omega}_{1}$ is defined by solving the problem (22b), and the problem (22a) is solved with a given stiffness orientation.

In the next sections, we recall first the structural stiffness (only) optimization algorithm (section 5.1), that we will next generalise to structural stiffness and strength optimization, considering the cases for which the leading objective is alternatively stiffness (section 5.2) or strength (section 5.3).

\subsection{Structural Rigidity Optimization}

The objective function is the compliance. Using eq. (20) and the complementary energy theorem, the optimization problem reads (S.A. stands for statically admissible):

$$
\min _{\left\{\bar{\Phi}_{1}, \bar{R}_{0 K}, \bar{R}_{1}\right\}} \min _{\left\{\mathbf{N}^{*} S . A .\right\}} \frac{1}{h} \int_{S_{p}} \mathbf{N}^{*} \cdot \mathbb{A}^{-1}\left(\bar{\Phi}_{1}, \bar{R}_{0 K}, \bar{R}_{1}\right) \mathbf{N}^{*} d S
$$

along with the geometric constraints, eq. (8).

The optimization algorithm used to solve this problem is the alternate direction algorithm introduced by Allaire and Kohn [11, 12], and already used in [5] in a two-step approach for maximizing the stiffness of laminated plates. The algorithm is divided into the following phases:

1. Initialization: the stiffness distribution over the structure is initialized and a first finite element (FE) analysis is conducted.

2. Local minimization of the complementary energy density: local definition of a new anisotropic stiffness distribution $\left(\bar{\Phi}_{1}^{(n+1)}, \bar{R}_{0 K}^{(n+1)}, \bar{R}_{1}^{(n+1)}\right)$ minimizing the complemen- 
tary energy density for a fixed field $\mathbf{N}^{(n)}$ :

$$
\begin{aligned}
& \min _{\left\{\bar{\Phi}_{1}, \bar{R}_{0 K}, \bar{R}_{1}\right\}} \mathbf{N}^{(n)} \cdot \mathbb{A}^{-1}\left(\bar{\Phi}_{1}, \bar{R}_{0 K}, \bar{R}_{1}\right) \mathbf{N}^{(n)} \\
& \text { subject to }\left\{\begin{array}{l}
2\left(\frac{\bar{R}_{1}}{R_{1}}\right)^{2}-1 \leq \frac{\bar{R}_{0 K}}{(-1)^{K} R_{0}}, \\
\left|\bar{R}_{0 K}\right| \leq R_{0}, \\
\bar{R}_{1} \geq 0 .
\end{array}\right.
\end{aligned}
$$

The minimization problem (24) has an analytical solution detailed in Sec. 6.1.

3. Global minimization of the complementary energy for a fixed anisotropic stiffness distribution: definition of the in-plane forces $\left(\mathbf{N}^{(n+1)}\right)$ and strains $\left(\varepsilon^{(n+1)}\right)$ fields linked to the new stiffness distribution $\left(\bar{\Phi}_{1}^{(n+1)}, \bar{R}_{0 K}^{(n+1)}, \bar{R}_{1}^{(n+1)}\right)$ through a FE analysis.

The second and third phases are repeated in loop until convergence [12] (the convergence proof is not presented here for the sake of shortness, but it is very similar to the convergence proof of the a priori algorithm presented in section 5.3).

\subsection{Structural Rigidity Optimization with A Posteriori Local Maximization of Strength}

Considering stiffness as the leading objective, the local minimization of the complementary energy (24) is performed with respect to $\bar{\Phi}_{1}, \bar{R}_{0 K}$ and $\bar{R}_{1}$, while the local minimization of the homogenized failure index along with the geometrical bounds of eq. (17), is performed with respect to $\bar{\Lambda}_{0 L}$ and $\bar{\Lambda}_{1}$ and with a known orthotropy direction, being $\bar{\Omega}_{1}=\bar{\Phi}_{1}$ or $\bar{\Phi}_{1} \pm \pi / 2$ :

$$
\begin{aligned}
& \min _{\left\{\bar{\Omega}_{1}, \bar{\Lambda}_{0 L}, \bar{\Lambda}_{1}\right\}} F_{H i l l}^{\text {Lam }}\left(\bar{\Omega}_{1}, \bar{\Lambda}_{0 L}, \bar{\Lambda}_{1}\right), \\
& \text { subject to }\left\{\begin{array}{l}
\bar{\Omega}_{1} \in\left\{\bar{\Phi}_{1}, \bar{\Phi}_{1} \pm \pi / 2\right\}, \\
2\left(\frac{\bar{\Lambda}_{1}}{\Lambda_{1}}\right)^{2}-1 \leq \frac{\bar{\Lambda}_{0 L}}{(-1)^{L} \Lambda_{0}}, \\
\left|\bar{\Lambda}_{0 L}\right| \leq \Lambda_{0}, \\
\bar{\Lambda}_{1} \geq 0 .
\end{array}\right.
\end{aligned}
$$

The minimization problem (25) has an analytical solution detailed in Sec. 6.2. 
At the structural optimization step, the laminate polar parameters $\bar{R}_{0 K}, \bar{R}_{1}, \bar{\Lambda}_{0 L}$ and $\bar{\Lambda}_{1}$ are assumed to be independent. The local minimization of the failure index is thus performed after the convergence of the structural rigidity optimization algorithm presented in the previous section, and this proves the convergence of this last algorithm with a posteriori maximization of strength.

This procedure hence leads to the anisotropic strength distribution $\left(\bar{\Lambda}_{0 L}^{o p t}, \bar{\Lambda}_{1}^{o p t}\right)$ that minimizes the failure index $F_{H i l l}^{\text {Lam }}$.

\subsection{Structural Rigidity Optimization with A Priori Local Maximization of Strength}

Considering strength as the leading objective, the local minimization of the homogenized failure index along with the geometrical bounds of eq. (17), is performed with respect to $\bar{\Omega}_{1}, \bar{\Lambda}_{0 L}$ and $\bar{\Lambda}_{1}$ :

$$
\begin{aligned}
& \min _{\left\{\bar{\Omega}_{1}, \bar{\Lambda}_{0 L}, \bar{\Lambda}_{1}\right\}} F_{\text {Hill }}^{\text {Lam }}\left(\bar{\Omega}_{1}, \bar{\Lambda}_{0 L}, \bar{\Lambda}_{1}\right), \\
& \text { subject to }\left\{\begin{array}{l}
2\left(\frac{\bar{\Lambda}_{1}}{\Lambda_{1}}\right)^{2}-1 \leq \frac{\bar{\Lambda}_{0 L}}{(-1)^{L} \Lambda_{0}}, \\
\left|\bar{\Lambda}_{0 L}\right| \leq \Lambda_{0}, \\
\bar{\Lambda}_{1} \geq 0 .
\end{array}\right.
\end{aligned}
$$

The minimization problem (26) has an analytical solution detailed in section 6.4.

On the other hand, the local minimization of the complementary energy along with the geometrical bounds of eq. (8), is performed with respect to $\bar{R}_{0 K}$ and $\bar{R}_{1}$ and with a known orthotropy direction, being now $\bar{\Phi}_{1}=\bar{\Omega}_{1}$ or $\bar{\Omega}_{1} \pm \pi / 2$ :

$$
\begin{aligned}
& \min _{\left\{\bar{\Phi}_{1}, \bar{R}_{0 K}, \bar{R}_{1}\right\}} \mathbf{N}^{(n)} \cdot \mathbb{A}^{-1}\left(\bar{\Phi}_{1}, \bar{R}_{0 K}, \bar{R}_{1}\right) \mathbf{N}^{(n)} \\
& \text { subject to }\left\{\begin{array}{l}
\bar{\Phi}_{1} \in\left\{\bar{\Omega}_{1}, \bar{\Omega}_{1} \pm \pi / 2\right\} \\
2\left(\frac{\bar{R}_{1}}{R_{1}}\right)^{2}-1 \leq \frac{\bar{R}_{0 K}}{(-1)^{K} R_{0}}, \\
\left|\bar{R}_{0 K}\right| \leq R_{0}, \\
\bar{R}_{1} \geq 0 .
\end{array}\right.
\end{aligned}
$$


At the structural optimisation step, the laminate polar parameters $\bar{R}_{0 K}, \bar{R}_{1}, \bar{\Lambda}_{0 L}$ and $\bar{\Lambda}_{1}$ are assumed to be independent. The local minimizations of the local complementary energy are performed after the definition of the orthotropy direction through the local minimization of the homogenised failure index. This leads to the loss of monotonic convergence in terms of global complementary energy, i.e. of the compliance.

In order to preserve the monotonic convergence of the numerical algorithm in terms of compliance, a constraint on the admissible orthotropy direction $\bar{\Omega}_{1}$ is added to the local minimization problem (26) of the homogenized failure index at each iteration $(n)$ :

$$
\begin{aligned}
& \min _{\left\{\bar{R}_{0 K}, \bar{R}_{1}\right\}}\left[\mathbf{N}^{(n)} \cdot \mathbb{A}^{-1}\left(\bar{\Omega}_{1}, \bar{R}_{0 K}, \bar{R}_{1}\right) \mathbf{N}^{(n)}\right] \leq \mathbf{N}^{(n)} \cdot \mathbb{A}^{-1}\left(\bar{\Phi}_{1}^{(n)}, \bar{R}_{0 K}^{(n)}, \bar{R}_{1}^{(n)}\right) \mathbf{N}^{(n)}, \\
& \text { subject to }\left\{\begin{array}{l}
2\left(\frac{\bar{R}_{1}}{R_{1}}\right)^{2}-1 \leq \frac{\bar{R}_{0 K}}{(-1)^{K} R_{0}}, \\
\left|\bar{R}_{0 K}\right| \leq R_{0}, \\
\bar{R}_{1} \geq 0
\end{array}\right.
\end{aligned}
$$

This constraint imposes a stagnation or a reduction of the local complementary energy at each iteration, and thus leads to the monotonic convergence in terms of compliance of the numerical algorithm. Let us hence consider the numerical procedure, whose convergence proof is given at the end of the section.

Numerical procedure (NP): problem defined by eqs. (26), (27) and (28) is solved numerically through the following steps:

1. Initialization: the stiffness distribution over the structure is initialized and a first finite element (FE) analysis is conducted. 
2. Local minimization of the failure index: local definition of a new anisotropic strength distribution $\left(\bar{\Omega}_{1}^{(n+1)}, \bar{\Lambda}_{0 K}^{(n+1)}, \bar{\Lambda}_{1}^{(n+1)}\right)$ minimizing the failure index for a fixed strain field:

$$
\begin{aligned}
& \qquad \min _{\left\{\bar{\Omega}_{1}, \bar{\Lambda}_{0 L}, \bar{\Lambda}_{1}\right\}} F_{H i l l}^{\operatorname{Lam}}\left(\bar{\Omega}_{1}, \bar{\Lambda}_{0 L}, \bar{\Lambda}_{1}\right), \\
& \text { subject to }\left\{\begin{array}{l}
(28), \\
2\left(\frac{\bar{\Lambda}_{1}}{\Lambda_{1}}\right)^{2}-1 \leq \frac{\bar{\Lambda}_{0 L}}{(-1)^{L} \Lambda_{0}}, \\
\left|\bar{\Lambda}_{0 L}\right| \leq \Lambda_{0}, \\
\bar{\Lambda}_{1} \geq 0 .
\end{array}\right.
\end{aligned}
$$

The numerical procedure used to solve this problem (29) is presented in section (6.4)

3. Local minimization of the complementary energy density with a known orthotropy direction, (27): local definition of a new anisotropic stiffness distribution, i.e. of $\left(\bar{\Phi}_{1}^{(n+1)}, \bar{R}_{0 K}^{(n+1)}\right.$, $\bar{R}_{1}^{(n+1)}$ ), minimizing the complementary energy density for a fixed field $\mathbf{N}^{(n)}$. The numerical procedure used to solve this problem (27) is presented in section (6.3).

4. Global minimization of the complementary energy for a fixed anisotropic stiffness distribution: definition of the in-plane forces $\left(\mathbf{N}^{(n+1)}\right)$ and strains $\left(\varepsilon^{(n+1)}\right)$ fields linked to the new stiffness distribution $\left(\bar{\Phi}_{1}^{(n+1)}, \bar{R}_{0 K}^{(n+1)}, \bar{R}_{1}^{(n+1)}\right)$ through a FE analysis.

The three last steps are repeated until convergence.

Some remarks on this numerical procedure, i.e. the algorithm a priori, are mandatory. It must be pointed out that, in this algorithm, only the monotonic decrease of the complementary energy is ensured. At each iteration of the algorithm, we locally maximize the strength and favour this strength maximisation with respect to the local complementary energy minimization. Nevertheless, once the strain and stress fields are updated, we have no insurance that the failure index of the laminate decreases and it may locally increase : the value of $F_{\text {Hill }}^{\text {Lam }}$ can oscillate along the iterations.

Let us now prove that for this numerical procedure, i.e. the algorithm a priori, the complementary energy is not increasing during iterations.

Proposition 5.1: for the numerical procedure NP described above for solving the problem 
defined by eqs. (26), (27) and (28), the complementary energy

$$
\int_{S_{p}} \mathbf{N} \cdot \mathbb{A}^{-1}\left(\bar{\Phi}_{1}, \bar{R}_{0 K}, \bar{R}_{1}\right) \mathbf{N} d S
$$

does not increase along the iterations:

$$
\begin{aligned}
\int_{S_{p}} \mathbf{N}^{(n+1)} \cdot \mathbb{A}^{-1}\left(\bar{\Phi}_{1}^{(n+1)}, \bar{R}_{0 K}^{(n+1)}, \bar{R}_{1}^{(n+1)}\right) \mathbf{N}^{(n+1)} d S & \\
& \leq \int_{S_{p}} \mathbf{N}^{(n)} \cdot \mathbb{A}^{-1}\left(\bar{\Phi}_{1}^{(n)}, \bar{R}_{0 K}^{(n)}, \bar{R}_{1}^{(n)}\right) \mathbf{N}^{(n)} d S \forall n .
\end{aligned}
$$

Proof : let us consider the anisotropic stiffness and strength distribution $\left(\bar{R}_{0 K}^{(n)}, \bar{R}_{1}^{(n)}, \bar{\Phi}_{1}^{(n)}\right.$, $\left.\bar{\Lambda}_{0 K}^{(n)}, \bar{\Lambda}_{1}^{(n)}, \bar{\Omega}_{1}^{(n)}\right)$ and the in-plane forces $\mathbf{N}^{(n)}$ and strain $\varepsilon^{(n)}$ fields at the iteration $(n)$.

The local minimization of the laminate failure index $F_{H i l l}^{\text {Lam }}$ consists in finding the strength parameters $\bar{\Lambda}_{0 K}^{(n+1)}, \bar{\Lambda}_{1}^{(n+1)}$ and $\bar{\Omega}_{1}^{(n+1)}$ solution to the optimization problem (29) at each point of the structure, with $\bar{\Omega}_{1}$ constrained by the condition (28) that is, at least, satisfied for $\bar{\Omega}_{1}=\bar{\Phi}_{1}^{(n)}$. The local minimization of the complementary energy is performed in order to find the new stiffness parameters solution of the optimization problem (27). Considering that $\bar{\Omega}_{1}^{(n+1)}$ determined through the local minimization of $F_{H i l l}^{\text {Lam }}$ satisfies the condition (28), we get:

$$
\int_{S_{p}} \mathbf{N}^{(n)} \cdot \mathbb{A}^{-1}\left(\bar{\Phi}_{1}^{(n+1)}, \bar{R}_{0 K}^{(n+1)}, \bar{R}_{1}^{(n+1)}\right) \mathbf{N}^{(n)} d S \leq \int_{S_{p}} \mathbf{N}^{(n)} \cdot \mathbb{A}^{-1}\left(\bar{\Phi}_{1}^{(n)}, \bar{R}_{0 K}^{(n)}, \bar{R}_{1}^{(n)}\right) \mathbf{N}^{(n)} d S
$$

The global minimization consists in determining the in-plane forces $\mathbf{N}^{(n+1)}$ and strain $\varepsilon^{(n+1)}$ fields, solution to the elastic problem linked to the stiffness distribution $\left(\bar{\Phi}_{1}^{(n+1)}, \bar{R}_{0 K}^{(n+1)}, \bar{R}_{1}^{(n+1)}\right)$. Thanks to the complementary energy theorem, the following inequality

$$
\begin{aligned}
\int_{S_{p}} \mathbf{N}^{(n+1)} \cdot \mathbb{A}^{-1}\left(\bar{\Phi}_{1}^{(n+1)}, \bar{R}_{0 K}^{(n+1)},\right. & \left.\bar{R}_{1}^{(n+1)}\right) \mathbf{N}^{(n+1)} d S \\
& \leq \int_{S_{p}} \mathbf{N}^{(n)} \cdot \mathbb{A}^{-1}\left(\bar{\Phi}_{1}^{(n+1)}, \bar{R}_{0 K}^{(n+1)}, \bar{R}_{1}^{(n+1)}\right) \mathbf{N}^{(n)} d S
\end{aligned}
$$


is verified. Considering eq. (32) and eq. (33), we get:

$$
\begin{aligned}
\int_{S_{p}} \mathbf{N}^{(n+1)} \cdot \mathbb{A}^{-1}\left(\bar{\Phi}_{1}^{(n+1)}, \bar{R}_{0 K}^{(n+1)}, \bar{R}_{1}^{(n+1)}\right) & \mathbf{N}^{(n+1)} d S \\
& \leq \int_{S_{p}} \mathbf{N}^{(n)} \cdot \mathbb{A}^{-1}\left(\bar{\Phi}_{1}^{(n)}, \bar{R}_{0 K}^{(n)}, \bar{R}_{1}^{(n)}\right) \mathbf{N}^{(n)} d S
\end{aligned}
$$

SO

$$
W_{c}^{(n+1)} \leq W_{c}^{(n)}
$$

Being $W_{c}$ a positive quantity that reduces at each iteration the monotonic convergence of the algorithm in terms of compliance is proved.

Since $W_{c}$ reduces at each iteration and converges, $F_{H i l l}^{\text {Lam }}$ will converge, though not necessarily monotonically, to a constant value due to the convergence of the strain field.

\section{Structural Optimization : Local Minimization Solutions}

\subsection{Algorithm A Posteriori: Local Complementary Energy Minimization}

The analytical resolution to problem (24), introduced in sections (5.1) and (5.2), was extensively studied in $[5,22,23]$. Nevertheless in those works, the geometric constraint on the polar parameters was not taken into account: only the thermodynamic constraints on the polar moduli were considered. The results taking into account for the geometric constraints for a basic ply with $K=0$ are summarized in Tab. 1 (a partial proof for the case of a basic ply with $K=0$ is presented in [22]). The complete proof for $K=0$ and $K=1$ is much more articulated than the proof presented in [22] and it is at present submitted in an other publication. Results of Tab. 1 show that the optimal values of the polar parameters of $\mathbb{A}$ depend upon the polar parameters of the in-plane forces $\mathbf{N}$ ( $T$ and $R$ are the spherical and deviatoric parts of the tensor, respectively, while $\Phi$ represents the direction of the first principal component of $\mathbf{N}$ ) and upon the direction of its principal components $N_{I}$ and $N_{I I}$. 


\begin{tabular}{cccc}
\hline & $(-\mathbf{1})^{\overline{\mathbf{K}}^{\text {opt }}} \overline{\mathbf{R}}_{\mathbf{0}}^{\text {opt }}$ & $\overline{\mathbf{R}}_{\mathbf{1}}^{\text {opt }}$ & $\bar{\Phi}_{\mathbf{1}}^{\text {opt }}$ \\
\hline $0 \leq \frac{R}{|T|} \leq \frac{R_{1}}{T_{1}}$ & {$\left[R_{0}\left(2\left(\frac{R T_{1}}{T R_{1}}\right)^{2}-1\right) ; R_{0}\right]$} & $T_{1} \frac{R}{|T|}$ & $\operatorname{direction}\left(\max \left(\left|N_{I}\right|,\left|N_{I I}\right|\right)\right)$ \\
$\frac{R_{1}}{T_{1}} \leq \frac{R}{|T|} \leq \frac{T_{0}+R_{0}}{2 R_{1}}$ & $R_{0}$ & $R_{1}$ & $\operatorname{direction}\left(\max \left(\left|N_{I}\right|,\left|N_{I I}\right|\right)\right)$ \\
$\frac{R}{|T|} \geq \frac{T_{0}+R_{0}}{2 R_{1}}$ & $R_{0}$ & $\frac{T_{0}+R_{0}}{2(R /|T|)}$ & $\operatorname{direction}\left(\max \left(\left|N_{I}\right|,\left|N_{I I}\right|\right)\right)$ \\
\hline
\end{tabular}

Table 1 Optimal values of stiffness polar parameters to maximize the plate stiffness for a basic ply with $K=0$.

6.2 Algorithm A Posteriori: Minimization of the Homogenized Failure Index with a Given Orthotropy Direction

In this section, we present the analytical solution to the local minimization problem (25) introduced in section (5.2) (a complete proof of the results can be found in [21]). This problem is solved in both cases $\bar{\Omega}_{1}=\bar{\Phi}_{1}^{\text {opt }}$ and $\bar{\Omega}_{1}=\bar{\Phi}_{1}^{\text {opt }} \pm \pi / 2$. The orientation with the lowest value of the objective function will be the optimal one. In addition, the admissible values of $\bar{\Lambda}_{0 L}$ and $\bar{\Lambda}_{1}$ change together with the value of the orthotropy shape parameter $L$ of the basic ply, see eq. (17). The resolution is split into two main groups, depending on the value $L$ of the basic ply. A complete summary of the solutions of the local minimization of $F_{\text {Hill }}^{\text {Lam }}$ is given in Tab. 2 for $L=0$ (a similar result exists for $L=1$ [21]). Thanks to the periodicity of the solution, the results are restricted to the range $\bar{\Omega}_{1}-\varphi=\left\{0 ; \frac{\pi}{2}\right\}$ (recalling that $\varphi$ is the direction of the first principal component of $\varepsilon$ ).

6.3 Algorithm A Priori: Local Complementary Energy Minimization with a Given Orthotropy Direction

The objective function of the optimization problem (27) introduced in section (5.3) reads:

$$
\begin{aligned}
& \mathbf{N}^{(n)} \cdot \mathbb{A}^{-1}\left(\bar{R}_{0 K}, \bar{R}_{1}\right) \mathbf{N}^{(n)}=\frac{1}{\Delta}\left[2\left(T_{0} T_{1}-\bar{R}_{1}^{2}\right) R^{2}+\left(T_{0}^{2}-\bar{R}_{0 K}^{2}\right) T^{2}\right. \\
+ & \left.2\left(\bar{R}_{1}^{2}-T_{1} \bar{R}_{0 K}\right) R^{2} \cos 4\left(\bar{\Phi}_{1}^{\text {opt }}-\Phi\right)+-4 \bar{R}_{1}\left(T_{0}-(-1)^{\bar{K}} \bar{R}_{0 K}\right) T R \cos 2\left(\bar{\Phi}_{1}^{\text {opt }}-\Phi\right)\right]
\end{aligned}
$$




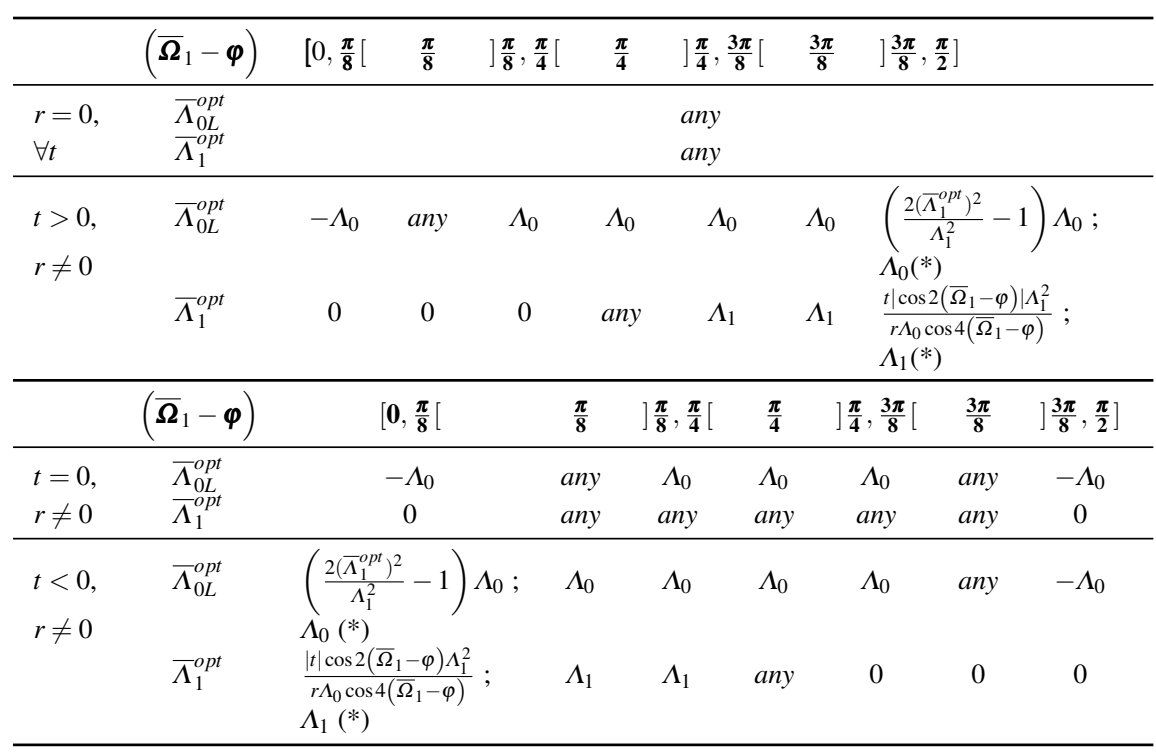

(*) the first solution is valid for $\frac{|t|}{r}<\frac{\Lambda_{0} \cos 4\left(\bar{\Omega}_{1}-\varphi\right)}{\Lambda_{1}\left|\cos 2\left(\bar{\Omega}_{1}-\varphi\right)\right|}$

Table 2 Solutions for $L=0$, local minimization of $F_{H i l l}^{\text {Lam }}$ with an imposed value of $\bar{\Omega}_{1}$.

in which $\bar{\Phi}_{1}^{o p t}=\bar{\Omega}_{1}^{\text {opt }}$ or $\bar{\Omega}_{1}^{o p t} \pm \pi / 2\left(\bar{\Omega}_{1}^{\text {opt }}\right.$ being determined through the local minimization of $\left.F_{H i l l}^{\text {Lam }}\right)$ and $\Delta=4 T_{1}\left(T_{0}^{2}-\bar{R}_{0 K}^{2}\right)-8 \bar{R}_{1}^{2}\left(T_{0}-\bar{R}_{0 K}\right)$. Problem (27) is solved in both cases of $\bar{\Phi}_{1}^{\text {opt }}=\bar{\Omega}_{1}^{\text {opt }}$ and $\bar{\Phi}_{1}^{\text {opt }}=\bar{\Omega}_{1}^{\text {opt }} \pm \pi / 2$, the orientation with the lowest value of the objective function is the optimal one. In this case, the derivatives with respect to $\bar{R}_{0 K}$ and $\bar{R}_{1}$ are rational functions of second degree polynomials in terms of $\bar{R}_{0 K}$ and $\bar{R}_{1}$. Therefore the minimum of the local complementary energy is searched numerically, using a fixed point algorithm (more details can be found in [21]).

6.4 Algorithm A Priori: Minimization of the Homogenized Failure Index

In this section we present the numerical resolution to the local minimization problem (29) introduced in section (5.3).

The optimization problem (26) has been solved analytically through two consecutive steps: first the minimization with respect to the orientation $\bar{\Omega}_{1}$ of the orthotropy axis and then, the minimization with respect to $\bar{\Lambda}_{0 L}$ and $\bar{\Lambda}_{1}$. The optimal orientation $\bar{\Omega}_{1}^{\text {opt }}$ has been 


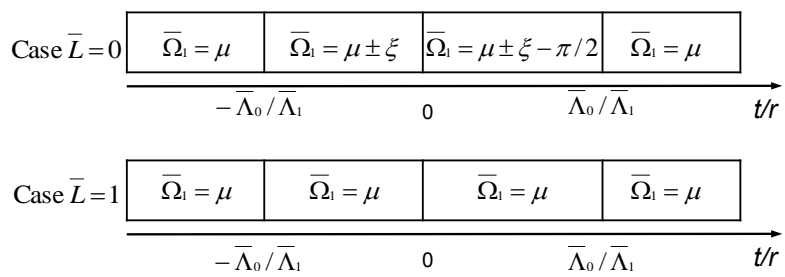

Fig. 1 Optimal orthotropy orientation to minimise $F_{H i l l}^{\text {Lam }}$.

evaluated following the same procedure already used in [20] where the problem of minimizing the failure index of a simple ply has been solved varying only the orthotropy orientation $\Omega_{1}$. Thus, we present directly the results in Fig. 1 where

$$
\mu=\operatorname{dir}\left(\min \left\{\left|\varepsilon_{I}\right|,\left|\varepsilon_{I I}\right|\right\}\right), \quad \xi=\frac{1}{2} \arccos \left(-\frac{\bar{\Lambda}_{1} t}{\bar{\Lambda}_{0 L} r}\right)
$$

Fig. 1 shows that the orthotropy shape parameter $\bar{L}$ of the plate plays a decisive role in the evaluation of the optimal orthotropy orientation. We have, thus, two types of solutions concerning the optimal orthotropy orientation: a solution that does not include the term $\xi$, that we will call solution non- $\xi$ and a solution including the term $\xi$, that we will call solution $\xi$. Therefore, in the second minimization phase, we have to minimize two different functions with respect to $\bar{\Lambda}_{0 L}$ and $\bar{\Lambda}_{1}$ :

$$
\begin{aligned}
& \text { solution non- } \xi: F_{H i l l}^{\text {Lam }}\left(\bar{\Lambda}_{0 L}, \bar{\Lambda}_{1}, \bar{\Omega}_{1}^{\text {opt }}\right)=4 r^{2} \Gamma_{0}+8 t^{2} \Gamma_{1}+4 \bar{\Lambda}_{0 L} r^{2}-16|t| r \bar{\Lambda}_{1} ; \\
& \text { solution } \xi: F_{H i l l}^{\text {Lam }}\left(\bar{\Lambda}_{0 L}, \bar{\Lambda}_{1}, \bar{\Omega}_{1}^{\text {opt }}\right)=4 r^{2} \Gamma_{0}+8 t^{2} \Gamma_{1}-4 \bar{\Lambda}_{0} r^{2}-8 t^{2} \bar{\Lambda}_{1}^{2}
\end{aligned}
$$

The optimal value of $F_{H i l l}^{\text {Lam }}$ obtained minimizing eq. (38) will be, then, compared with that obtained minimizing eq. (39). The solution that gives the minimum value of $F_{H i l l}^{\text {Lam }}$ will be the global optimal solution of problem (26). For the sake of brevity, the solutions of this case are directly summarized in Tab. 3, while the extended proof is reported in [21]. The first row of Tab. 3 gives the optimal value of $\bar{\Omega}_{1}$, while the rows below report the optimal values of the polar moduli $\bar{\Lambda}_{0 L}$ and $\bar{\Lambda}_{1}$. In particular, the result shown in the first row of Tab. 3 leads to an important consequence: we know from Tab. 1 that the optimal orthotropy orientation that maximizes the stiffness is always aligned with the directions of principal 
stresses. On the other hand, Tab. 3 shows that the optimal orthotropy orientation to maximize the strength is always aligned with the direction of the principal strains. Therefore, if in an anisotropic structure the principal stress and strain are aligned, the optimal orthotropy orientation maximizing the strength will be the same one maximizing the stiffness.

\begin{tabular}{llcll}
\hline & & $\bar{\Omega}_{\mathbf{1}}^{\text {opt }}=\operatorname{dir}\left(\min \left\{\left|\varepsilon_{\mathbf{I}}\right|,\left|\varepsilon_{\mathbf{I I}}\right|\right\}\right)$ & $\forall \boldsymbol{L}, \boldsymbol{t}, \boldsymbol{r}$ \\
\hline \hline & $\overline{\boldsymbol{\Lambda}}_{\mathbf{0 L}}^{\text {opt }}$ & $\overline{\boldsymbol{\Lambda}}_{\mathbf{1}}^{\text {opt }}$ & $\mathbf{F}_{\text {Hill }}^{\text {Lam }(\mathbf{o p t})}$ \\
\hline$L=0,1$ & $r=0, t \neq 0$ & any & any & $8 t^{2} \Gamma_{1}$ \\
\hline \multirow{2}{*}{$L=0$} & $\frac{|t|}{r} \leq \frac{\Lambda_{0}}{\Lambda_{1}}$ & $\left(\frac{2 t^{2} \Lambda_{1}^{2}}{r^{2} \Lambda_{0}^{2}}-1\right) \Lambda_{0}$ & $\frac{|t| \Lambda_{1}^{2}}{r \Lambda_{0}}$ & $4 r^{2} \Gamma_{0}+8 t^{2} \Gamma_{1}-4 r^{2} \Lambda_{0}-8 t^{2} \frac{\Lambda_{1}^{2}}{\Lambda_{0}}$ \\
& $\frac{|t|}{r} \geq \frac{\Lambda_{0}}{\Lambda_{1}}$ & $\Lambda_{0}$ & $\Lambda_{1}$ & $4 r^{2} \Gamma_{0}+8 t^{2} \Gamma_{1}+4 \Lambda_{0} r^{2}-16|t| r \Lambda_{1}$ \\
& $t=0, r \neq 0$ & $-\Lambda_{0}$ & 0 & $4 r^{2} \Gamma_{0}-4 \Lambda_{0} r^{2}$ \\
\hline$L=1$ & $t \neq 0, r \neq 0$ & $-\Lambda_{0}$ & $\Lambda_{1}$ & $4 r^{2} \Gamma_{0}+8 t^{2} \Gamma_{1}-4 \Lambda_{0} r^{2}-16|t| r \Lambda_{1}$ \\
& $t=0, r \neq 0$ & $-\Lambda_{0}$ & {$\left[0, \Lambda_{1}\right]$} & $4 r^{2} \Gamma_{0}-4 \Lambda_{0} r^{2}$ \\
\hline
\end{tabular}

Table 3 Global solution of the local minimization of $F_{H i l l}^{\text {Lam }}$ including $\bar{\Omega}_{1}$ as optimization parameter.

However, if this analytical solution does not satisfy the constraint (28) the optimization problem (29) is solved into an admissible set of intervals. This admissible set is obtained in a two step procedure. First, the optimization problem (27) is solved numerically (see section 6.3) for values of $\bar{\Phi}_{1} \in\left[-\frac{\pi}{2} ; \frac{\pi}{2}\right]$ using a coarse discretization step . Then the admissible bounds are precisely calculated by refining the discretization step around the previous coarse bounds.

Once the admissible set of $\bar{\Omega}_{1}$ (formally written $\left[\bar{\Omega}_{1 A} ; \bar{\Omega}_{1 B}\right]$ ) is obtained, the optimization problem (26) is solved analytically (see section 6.2) for values of $\bar{\Omega}_{1} \in\left[\bar{\Omega}_{1 A} ; \bar{\Omega}_{1 B}\right]$ using a coarse discretization step. If the coarse optimal value of $\bar{\Omega}_{1}$ is admissible, a refinement of the discretization step is performed to obtain a precise value for $\bar{\Omega}_{1}^{\text {opt }}$. If the coarse optimal value of $\bar{\Omega}_{1}$ is not admissible, the optimal value is chosen among the bounds of $\bar{\Omega}_{1}$ (i.e. $\bar{\Omega}_{1 A}$ or $\bar{\Omega}_{1 B}$ ). 


\section{Lay-Up Design}

The design process turns now on the definition of a stacking sequence satisfying the optimal distribution of polar parameters issued from the first step.

As discussed in Sec. 3, eqs. (7) and (16) show that the correspondence between the elastic behavior of a laminate and the stacks is not bijective. This means that it is possible to obtain the same mechanical parameters with several stacking sequences having the same number of identical plies. Moreover, the number of laminates having the same elastic behavior is extremely large and rapidly increases with the layers number, see [24].

The problem of designing laminates with given elastic properties as a global optimization problem, without restricting a priori assumptions on the stacking sequence, was formulated in a completely general way in the works of Vannucci et al. [24-26]. In the framework of the polar formalism, the problem is formulated as a minimum distance problem in the space of the polar parameters, the design variables being the plies orientations. The solution is given by a stacking sequence that reduces to zero the distance of its mechanical properties from the optimal ones found as solution of the structural optimization problem.

For the problem at hand, the optimization problem of the lay-up design phase is:

find, for a given set

$$
\left\{\bar{K}^{o p t}, \bar{R}_{0}^{o p t}, \bar{R}_{1}^{o p t}, \bar{L}^{o p t}, \bar{\Lambda}_{0}^{o p t}, \bar{\Lambda}_{1}^{o p t}, \bar{\Phi}_{1}^{o p t}\right\}
$$

a vector of plies orientations $\left(\delta_{1}, \delta_{2}, \ldots, \delta_{n}\right)$ such that:

$$
\left\{\begin{array}{l}
\bar{R}_{0}=\bar{R}_{0}^{o p t}, \\
\bar{R}_{1}=\bar{R}_{1}^{o p t}, \\
\bar{\Lambda}_{0}=\bar{\Lambda}_{0}^{o p t}, \\
\bar{\Lambda}_{1}=\bar{\Lambda}_{1}^{o p t}, \\
\bar{\Phi}_{0}-\bar{\Phi}_{1}=\bar{K}^{o p t} \pi / 4, \\
\bar{\Omega}_{0}-\bar{\Omega}_{1}=\bar{L}^{o p t} \pi / 4, \\
\bar{\Phi}_{1}-\bar{\Omega}_{1}=0 ; \pi / 2, \\
\bar{\Phi}_{1}=\bar{\Phi}_{1}^{o p t}, \\
\mathbb{B}=\mathbb{O} .
\end{array}\right.
$$


This problem needs to be solved at any point, i.e. for each finite element used to discretize the structure.

According to Vannucci, [25], we formalize problem (40) as follows:

$$
\min _{\delta_{k}} I\left(f_{i}\left(\delta_{k}\right)\right)=\sum_{i} f_{i}^{2}\left(\delta_{k}\right) \text { with } k=1,2, . ., n
$$

where each sub-objective $f_{i}\left(\delta_{k}\right)$ represents a requirement to be satisfied. In our case it is $n=9$ and $\left(\zeta=1\right.$ if $\bar{\Phi}_{1}\left(\delta_{k}\right)=\bar{\Omega}_{1}\left(\delta_{k}\right) \pm \pi / 2$, otherwise $\zeta=0$, see sect. (6.3)):

$$
\begin{gathered}
f_{1}\left(\delta_{k}\right)=\frac{\left|\bar{\Phi}_{0}\left(\delta_{k}\right)-\bar{\Phi}_{1}\left(\delta_{k}\right)\right|}{\pi / 4}-\bar{K}^{o p t}, f_{2}\left(\delta_{k}\right)=\frac{\left|\bar{\Omega}_{0}\left(\delta_{k}\right)-\bar{\Omega}_{1}\left(\delta_{k}\right)\right|}{\pi / 4}-\bar{L}^{o p t} \\
f_{3}\left(\delta_{k}\right)=\frac{\bar{\Omega}_{1}\left(\delta_{k}\right) \pm \zeta \pi / 2-\bar{\Phi}_{1}\left(\delta_{k}\right)}{\pi / 4}, f_{4}\left(\delta_{k}\right)=\frac{\bar{R}_{0}\left(\delta_{k}\right)-\bar{R}_{0}^{o p t}}{R_{0}} \\
f_{5}\left(\delta_{k}\right)=\frac{\bar{R}_{1}\left(\delta_{k}\right)-\bar{R}_{1}^{o p t}}{R_{1}}, f_{6}\left(\delta_{k}\right)=\frac{\bar{\Lambda}_{0}\left(\delta_{k}\right)-\bar{\Lambda}_{0}^{o p t}}{\Lambda_{0}} \\
f_{7}\left(\delta_{k}\right)=\frac{\bar{\Lambda}_{1}\left(\delta_{k}\right)-\bar{\Lambda}_{1}^{o p t}}{\Lambda_{1}}, f_{8}\left(\delta_{k}\right)=\frac{\left\|\mathbb{B}\left(\delta_{k}\right)\right\|}{\|\mathbb{Q}\|}, f_{9}\left(\delta_{k}\right)=\frac{\bar{\Phi}_{1}\left(\delta_{k}\right)-\bar{\Phi}_{1}^{o p t}}{\pi / 4}
\end{gathered}
$$

Hence, problem (40) can be formulated as the search for an absolute minimum, equal to zero, of the global objective function (41), composed by nine sub-objectives (42), each one representing one of the imposed requirements.

In the domain of the ply orientations $I\left(f_{i}\left(\delta_{k}\right)\right)$ is a highly non-convex function, see eqs. (7) and (16). The use of classical descent strategies is, hence, not recommended for the solution of such a problem for which the use of metaheuristics seems to be more indicated. Thanks to previous works on the matter [27, 28], we decided to solve the second step problem using the Genetic Algorithm (GA) BIANCA (BIological ANalysis of Composite Assemblages), originally developed by Vincenti et al. [29] since 2002. The main features of the GA BIANCA are described in [13, 29].

The evaluation of $\max \left(F_{H i l l}^{P l y}\right)$ discussed in Sec. 4, will be now very helpful to check for the first ply failure. After the structural optimization step we have $F_{H i l l}^{L a m}<1$ everywhere, but the calculation of $\max \left(F_{H i l l}^{P l y}\right)$ can lead to two different situations:

1. $\max \left(F_{\text {Hill }}^{P l y}\right)<1$, therefore, we will have no limits to impose on the plies orientations when searching the stacking sequence; 
2. $\max \left(F_{H i l l}^{P l y}\right) \geq 1 \Longrightarrow$ there can be some orientations $\Omega_{1}^{(k)}$ of the plies such that $F_{H i l l}^{P l y} \geq 1$. In this case, we look for the set of values of $\Omega_{1}^{(k)}$ where $F_{H i l l}^{P l y}\left(\Omega_{1}^{(k)}\right) \geq 1$; this set is not admissible for the ply orientation.

This set can be calculated solving eq. (18) $)_{2}$, reformulated as follows:

$$
\begin{aligned}
8(-1)^{L} \Lambda_{0} r^{2} \cos ^{2} 2\left(\Omega_{1}^{(k)}-\varphi\right)+16 t r \Lambda_{1} \cos 2\left(\Omega_{1}^{(k)}-\varphi\right)+ & \\
& +4 r^{2} \Gamma_{0}+8 t^{2} \Gamma_{1}-4(-1)^{L} \Lambda_{0} r^{2}-1 \geq 0 .
\end{aligned}
$$

\section{Numerical Example}

Let us consider an uncoupled and orthotropic square plate with a centered hole, subjected to biaxial in-plane loading conditions (Fig. 2). Only a quarter of the plate is discretized for symmetry reasons. The total thickness $h$ of the plate is fixed to $3.75 \mathrm{~mm}$ (that corresponds to a laminated plate composed by 30 identical plies of thickness $0.125 \mathrm{~mm}$ ). The loading values are $F_{x}=500 \mathrm{~N} / \mathrm{mm}$ and $F_{y}=250 \mathrm{~N} / \mathrm{mm}$. Both the stiffness and strength properties of the basic ply (Carbon-Epoxy T300-5208), along with the numerical values of the polar parameters, are given in Tab. 4. The initial values of the stiffness polar parameters are those of a cross-ply laminate $\left(\bar{R}_{0}^{(0)}=R_{0}\right.$ and $\left.\bar{R}_{1}^{(0)}=0\right)$ oriented along $x$ and $y$ axis over the whole plate. The initial strength polar parameters are chosen equal to those of the basic ply $\left(\bar{\Lambda}_{0 L}^{(0)}=\right.$ $\Lambda_{0}$ and $\left.\bar{\Lambda}_{1}^{(0)}=\Lambda_{1}\right)$.

\subsection{First step: Structural Optimization}

The main results of the structural optimization given by the two algorithms and their comparison with the initial condition are presented in Tab. 5. We present the values, at the initial and optimal conditions, of the compliance, the maximum laminate and ply failure indexes and the maximum displacement. For all these quantities, a sensible reduction is obtained.

According to the theory, the lowest value of the compliance is obtained with the algorithm a posteriori (Sec. 5.2), that favours the minimization of the complementary energy. 


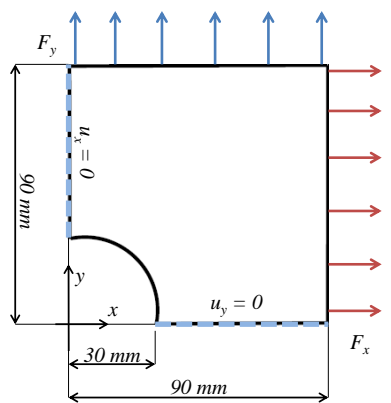

Fig. 2 Geometry and boundary conditions.

\begin{tabular}{|c|c|c|c|}
\hline \multicolumn{4}{|c|}{ Mechanical properties } \\
\hline \multicolumn{2}{|c|}{ Stiffness } & \multicolumn{2}{|c|}{ Strength } \\
\hline$E_{1}[\mathrm{MPa}]$ & 181000 & $X[\mathrm{MPa}]$ & 1500 \\
\hline$E_{2}[\mathrm{MPa}]$ & 10300 & $Y[\mathrm{MPa}]$ & 40 \\
\hline$G_{12}[\mathrm{MPa}]$ & 7170 & $S[\mathrm{MPa}]$ & 68 \\
\hline$v_{12}[\mathrm{MPa}]$ & 0.28 & & \\
\hline \multicolumn{4}{|c|}{ Polar parameters } \\
\hline \multicolumn{2}{|c|}{ Parameters of $\mathbb{Q}$} & \multicolumn{2}{|c|}{ Parameters of $\mathbb{G}$} \\
\hline$T_{0}[\mathrm{MPa}]$ & 26880 & $\Gamma_{0}$ & 11746 \\
\hline$T_{1}[\mathrm{MPa}]$ & 24744 & $\Gamma_{1}$ & 15461 \\
\hline$R_{0}[\mathrm{MPa}]$ & 19710 & $\Lambda_{0}$ & 628 \\
\hline$R_{1}[\mathrm{MPa}]$ & 21433 & $\Lambda_{1}$ & 5898 \\
\hline$K$ & 0 & $L$ & 0 \\
\hline
\end{tabular}

Table 4 Mechanical properties and polar parameters of the basic ply (Carbon-Epoxy T300-5208).

\begin{tabular}{llll}
\hline & Alg. posteriori & Alg. priori \\
\hline & $\begin{array}{l}\text { Initial } \\
\text { condition }\end{array}$ & $\begin{array}{l}\text { Optimal cond., } \\
\text { (\% reduction) }\end{array}$ & $\begin{array}{l}\text { Optimal cond., } \\
\text { (\% reduction) }\end{array}$ \\
\hline Compliance $[\mathrm{N} \mathrm{mm}]$ & 10232 & $6921(32.4)$ & $6931(32.2)$ \\
Max F Fam & 8.358 & $0.476(94.3)$ & $0.449(94.6)$ \\
Max F Fll & - & 0.476 & 0.459 \\
Max displacement $[\mathrm{mm}]$ & 0.317 & $0.138(56.5)$ & $0.139(56.1)$ \\
\hline
\end{tabular}

Table 5 Main results. 
The optimal value of the compliance when using the algorithm a priori (Sec. 5.3) is still significantly reduced with respect to the starting condition, but it is greater than the optimal value obtained using the algorithm a posteriori. The algorithm a priori shows a lower value of the maximum of failure index of the laminate $\left(F_{H i l l}^{\operatorname{Lam}}\right)$ with respect to that obtained with the algorithm a posteriori, and this result is explained by the fact that the algorithm a priori favours the minimization of $F_{H i l l}^{\text {Lam }}$ over that of the compliance.

The distribution, over the plate, of the local complementary energy $W_{c}$ in the initial and optimal configurations, using the two algorithms, is presented in Figs. 3: the two algorithms give a very similar distribution (Figs. 3(b) and (c)).

The distribution of $F_{H i l l}^{L a m}$, in the initial and optimal configurations for the two algorithms, is presented in Figs. 4: again, the two algorithms give a very similar distribution (Figs. 4(b) and (c)).

The evolution of the compliance along the iterations is presented in Fig. (5) (the initial value has been omitted in order to obtain a more precise view of the curves). The monotonic convergence, as theoretically proved, is clearly seen on those curves.

Finally, the distribution, for the algorithm a posteriori, of the optimal stiffness polar moduli $\bar{R}_{0 K}^{o p t}$ and $\bar{R}_{1}^{o p t}$, optimal orthotropy direction $\bar{\Phi}_{1}^{\text {opt }}$ and maximal failure index of the ply $\max \left(F_{H i l l}^{P l y}\right)$ are presented in Fig. (6), while the optimal polar strength moduli $\bar{\Lambda}_{0 L}^{\text {opt }}$ and $\bar{\Lambda}_{1}^{\text {opt }}$ are everywhere equal to those of the elementary ply, respectively (similar distributions are obtained for the a priori algorithm and are omitted for the sake of brevity). Although the maximal value of the ply failure index $\max \left(F_{H i l l}^{p l y}\right)$ may significantly differs from the value of the laminate failure index, their values are equal where $F_{H i l l}^{\text {Lam }}$ is maximal in the plate domain (see Tab. 5). This is explained by the fact that the optimal laminate is at this particular place a unidirectional one $\left(\bar{R}_{0 K}^{o p t}=R_{0}\right.$ and $\left.\bar{R}_{1}^{o p t}=R_{1}\right)$.

\subsection{Second Step: Lay-Up Design}

The problem of finding the stacking sequence for a generic element of the laminate has been solved using the genetic algorithm BIANCA [13, 28, 30, 31]. 

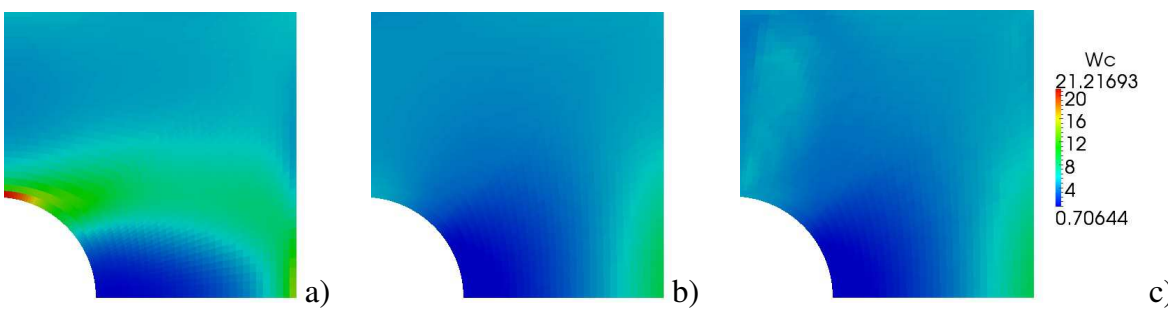

Fig. 3 Local complementary energy $\left(W_{C}\right)$ distribution for the initial (a) and optimal configurations using algorithm a posteriori (b) and algorithm a priori (c).
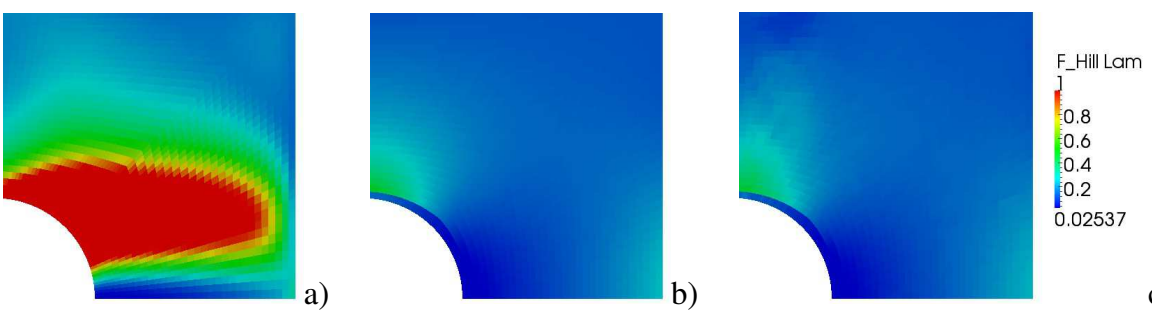

Fig. $4 F_{H i l l}^{\text {Lam }}$ distribution for the initial (a) and optimal configurations using algorithm a posteriori (b) and algorithm a priori (c).
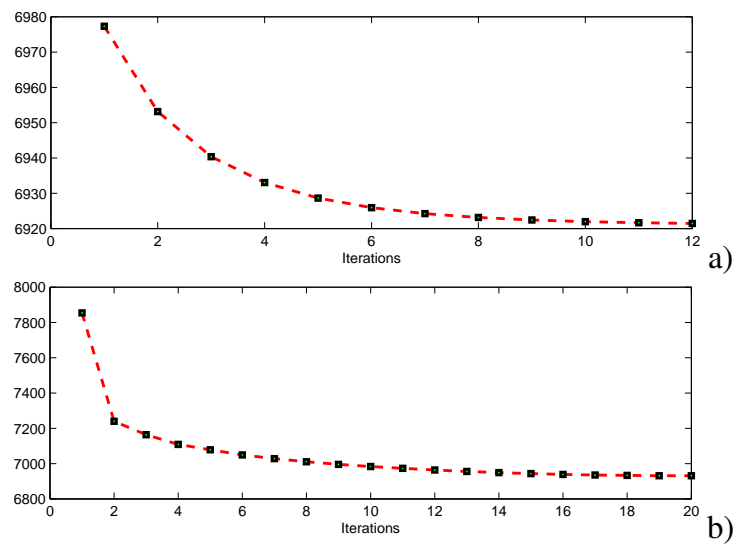

Fig. 5 Compliance of the plate along the iterations : algorithm a posteriori (a) and a priori (b). 
We consider the optimal anisotropy fields obtained by the a posteriori optimization algorithm (Fig. (6)). In this example, the maximal value of the failure index of the ply is smaller than 1 all over the plate, so all the angles are admissible for the ply orientations (see section 7).

Tab. 6 shows an example of stacking sequence found using the genetic algorithm together with the values of the partial objectives and of the global objective function $I\left(f_{i}\left(\delta_{k}\right)\right)$; exact solutions correspond to the zeroes of the partial objective functions. The resulting laminate is uncoupled (the value of the corresponding partial function varies from $0.37 \times 10^{-6}$ to $0.41 \times 10^{-3}$ ) and orthotropic (the value of the corresponding partial function is always about $10^{-7}$ ).

The same procedure is applied to all the finite elements discretizing the plate (1800 in this example). In Fig. 7(c), the value of final value of the total objective function $I\left(f_{i}\left(\delta_{k}\right)\right)$ for each element is presented. Figs. 7(a,b) show the optimal orientation of the first two plies composing the plate (the plate being composed by 30 plies, each elementary ply having a thickness of $0.125 \mathrm{~mm}$ ).

\section{Perspectives and Open Problems}

The true objective of this paper was to propose a new approach to the design of laminated structures having a variable anisotropy. So, we have chosen to concentrate our efforts on giving an as much as possible mathematically rigorous procedure and in showing that such a goal can be achieved.

Nevertheless, we have left apart some points that should be, to our opinion, investigated in the next future. Let us briefly list them:

- the failure criterion used here was the Tsai-Hill one but other criteria should also be considered. The global procedure is not affected by a change of the criterion, nevertheless this needs a particular attention in the theoretical developments given in section 4 . In fact, in criteria like those of Hoffmann or Tsai-Wu, the presence of a linear term besides the quadratic one gives supplementary terms in the expression of the failure index for both the ply and the laminate, while criteria not based upon energetic considerations, 

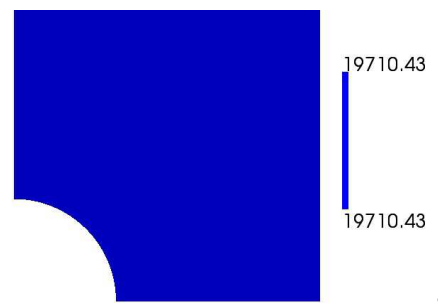

a)
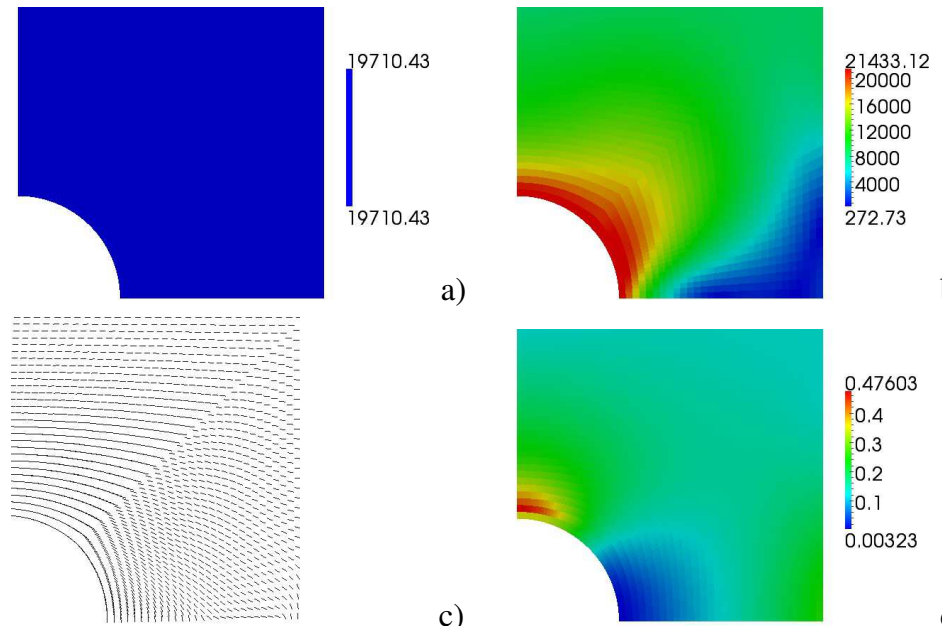

Fig. $6 \bar{R}_{0 K}^{\text {opt }}(\mathrm{a}), \bar{R}_{1}^{\text {opt }}$ (b), $\bar{\Phi}_{1}^{\text {opt }}$ (c) and $\max \left(F_{\text {Hill }}^{P l y}\right.$ ) (d) and for the optimal configuration, algorithm a posteriori.

\begin{tabular}{ccc}
\hline & Target values & Partial obj. funct. \\
\hline Uncoupling & $\mathbb{B}=0$ & $0.41 E-03$ \\
$\bar{R}_{0}[\mathrm{MPa}]$ & 19710.431 & $0.67 E-04$ \\
$\bar{R}_{1}[\mathrm{MPa}]$ & 8746.335 & $0.34 E-03$ \\
$\bar{\Lambda}_{0}$ & 628.713 & $0.11 E-02$ \\
$\bar{\Lambda}_{1}$ & 1998.75 & $0.65 E-04$ \\
$\bar{\Phi}_{0}-\bar{\Phi}_{1}$ & 0 & $0.15 E-06$ \\
$\bar{\Omega}_{0}-\bar{\Omega}_{1}$ & $\pi / 2$ & $0.15 E-06$ \\
$\bar{\Phi}_{1}-\bar{\Omega}_{1}$ & $\pi / 2$ & $0.15 E-06$ \\
\hline Total $\mathbf{o b j}$ funct. $I\left(f_{i}\left(\boldsymbol{\delta}_{k}\right)\right)=\mathbf{2 \times 1 0 ^ { - 3 }}$
\end{tabular}

Sequence $\quad 5 /-90 / 5 / 3 /-10 /-6 / 89 / 5 / 3 /-10 / 90 / 0 /-1 / 2 /-90$ /1/-7/1/-90/89/2/89/-3/2/90/-90/1/2/-3/1

Table 6 Stacking sequence design, example.
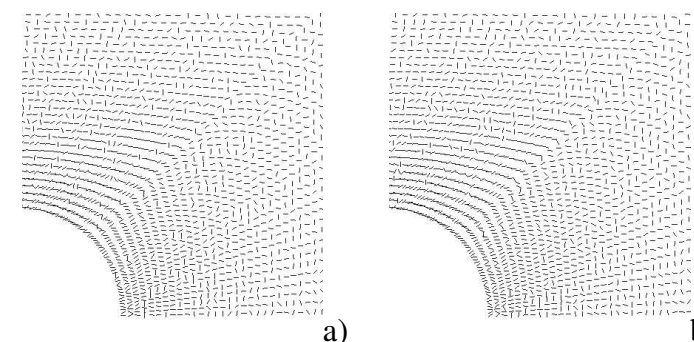

b)

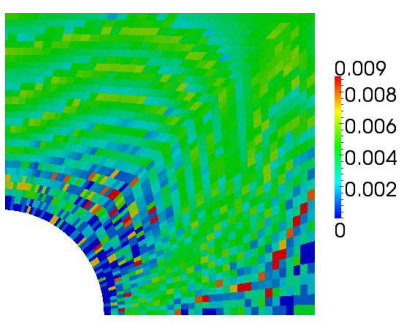

Fig. 7 Optimal fibers orientation in the two firsts plies $(a, b)$ and distribution of the total objective function (c). 
like the one of the maximum stress, are not defined by a unique, quadratic condition, but by five inequalities. The mathematical consequences of these facts should be attentively evaluated in view of the application of the entire procedure with other failure criteria;

- all the developments have been done for the only case of simple in-plane actions: the case of a pure bending state should also be considered, and this should not imply substantial modifications to the procedure. Nevertheless, it should be investigated what are the consequences of such a modification on the mathematical formulation and on the homogenized strength criterion;

- the previous point leads to a more complicate problem: to consider the case of contemporary in-plane and bending actions. In such a case we are faced to an open problem in laminates design: the exact analytical definition of the geometric bounds on the polar components for the laminate is not yet known when both extension and bending parameters, that are not independent, are taken into account simultaneously; this is a major problem in laminates optimization problems, and despite the attempts to solve it, done with the use of the so-called lamination parameters, that substantially coincide with the dimensionless polar parameters of the laminate, its solution remains an open problem. Another relevant point comes from the contemporary presence of actions of different nature: bending and extension. The way these actions interact, for both the stiffness and, mainly, for the strength optimization problem, is still not known;

- in order to take into account the singular stress concentration due to the interlaminar stresses near free edges, it is necessary to know the stacking sequence. Such a supplementary failure mode could be introduced in the second step of the procedure as a supplementary constraint for the genetic algorithm;

- we did not introduce supplementary constraints in the formulation of the optimization problem we dealt with. Nevertheless, such constraints are often present in a structural problem. Examples can be lower bounds on the fundamental vibration frequency or on the buckling load. Also, constraints whose origin is technological could be considered. It should be interesting to investigate how taking into account such constraints should 
modify the algorithms developed for the resolution of the structural optimization problems;

- the discretization made for the structure gives a solution that changes from an element to another one. This implies, namely, a change in the orthotropy direction when passing from an element to another aside: the orthotropy direction, and hence, finally, the fiber direction in a layer, is not continuous, generally speaking. Of course, this is not admissible in practice. Even more compelling is the fact that not only the direction of the fibers in a layer must be a continuous quantity, but it should also satisfy to some technological restrictions on the curvature: too sudden changes in the direction are not admissible. This geometrical problem of determining a technologically suitable and continuous fiber path corresponding to the optimal structural solution is a completely open problem; only a suitable resolution of this problem should really open the way to industrial applications of laminated structures with locally variable stiffness;

\section{Conclusions}

In this paper, we dealt with the problem of developing a new strategy for the optimal design of anisotropic laminated structures with respect to stiffness and strength. Some original and innovating aspects are contained in this paper, namely the fact that the general problem of determining the stiffest and strongest laminated structure has been formulated in a completely new way: thanks to the independence of the material properties determining stiffness and strength, we can introduce an optimization problem which is not a multiobjective one, nor a constrained one (in the sense that one of the two above properties constraints the other one).

Also, we have considered locally variable mechanical properties, so that we can, in a sense, qualify such a problem of anisotropy topology optimization: unlike usual topology optimization, where the shape has to be designed, here we want to determine the best distribution of the anisotropy properties, for both strength and stiffness, for a fixed shape. 
This new problem has been formulated adapting to this specific case an existing twostep approach; a first step, that we have called the structural problem, and a second one, called the lay-up problem.

The use of the polar formalism for the description of anisotropy has simplified both these problems, giving us, on one side, a reduction of the design variable number, on the other side, the analytical solution to some minimizations, which speeds up the numerical solution.

The analytical minimization of the strength local functional has brought also to an important theoretical result: when the tensors of stress and strain are coaxial, the optimal material orientation maximizing stiffness is the same that maximizes strength, so this solution represents a real global optimum for both stiffness and strength properties.

Acknowledgements: FNR of Luxembourg, supporting the first author through Aides à la Formation Recherche Grant PHD-09-184, is gratefully acknowledged.

\section{References}

1. Banichuk, N. V.: Problems and methods of optimal structural design. Plenum Press (1983).

2. Abrate, S.: Optimal design of laminates plates and shells. Composite Structures, 29,269-286 (1994).

3. Gürdal, Z., Haftka, R. T. and Hajela, P.: Design and optimization of laminated composite materials. Wiley-Interscience (1999).

4. Ghiasi, H., Pasini, D. and Lessard, L.: Optimum stacking sequence design of composite materials. part i: Constant stiffness design. Composite Structures, 90, 1-11 (2009).

5. Jibawy, A., Julien, C., Desmorat, B., Vincenti, A. and Léné, F.: Hierarchical structural optimization of laminated plates using polar representation. International Journal of Solids and Structures, 48, 2576-2584 (2011). 
6. Van Campen, J., Kassapoglou, C. and Gürdal, Z.: Generating realistic laminate fiber angle distributions for optimal variable stiffness laminates. Composites: part B, $\mathbf{4 3}$, 354-360 (2011).

7. De Leon, D. M. , de Souza, C. E., Fonseca, J. S. O. and Da Silva, R. G. A.: Aeroelastic tailoring using fiber orientation and topology optimization. Structural and Multidisciplinary Optimization, 46, 663-677 (2012).

8. Miki, M.: Material design of composite laminates with required in-plane elastic properties. In T. Hayashi, K. Kawata, and S. Umekawa, editors, Progress in Science and Engineering of Composites, ICCM-IV, pp. 1725-1731, Tokyo (1982).

9. Miki, M.: Design of laminated fibrous composite plates with required flexural stiffness. ASTM STP, 864, 387-400 (1985).

10. Vannucci, P.: A note on the elastic and geometric bounds for composite laminates. Journal of Elasticity, 112, 199-215 (2013).

11. Allaire, G. and Kohn, R.: Optimal design for minimum weight and compliance in plane stress using extremal micro structures. European Journal of Mechanics, A/Solids, 12, 839-878 (1993).

12. Allaire, G. and Kohn, R.: Shape optimization by the homogenization method. Numerische Mathematik, 76, 27-68 (1997).

13. Montemurro, M.: Optimal Design of Advanced Engineering Modular Systems through a New Genetic Approach. PhD thesis, University Paris 6, http://tel.archivesouvertes.fr/tel-00955533 (2012).

14. Villaggio, P.: Mathematical Models for Elastic Structures. Cambridge, The University Press (1997).

15. Hill, R.: A theory of the yielding and plastic flow of anisotropic metals. Proceedings of the Royal Society of London. Series A, Mathematical and Physical Sciences, 193, 281-297 (1948).

16. Jones, R.M.: Mechanics of composite materials. Taylor \& Francis (1999).

17. Verchery, G.: Les invariants des tenseurs d'ordre 4 du type de l'élasticité. In Proceeding of the Euromech Colloquium 115, vol. 115, pp. 93-104, Villard-de-Lans, France (1979). 
18. Vannucci, P.: Plane anisotropy by the polar method. Meccanica, 40, 437-454 (2005).

19. Vannucci, P.: The polar analysis of a third order piezoelecticity-like plane tensor. International Journal of Solids and Structures, 44, 7803-7815 (2007).

20. Catapano, A., Desmorat, B. and Vannucci, P.: Invariant formulation of phenomenological failure criteria for orthotropic sheets and optimisation of their strength. Mathematical Methods in Applied Sciences, 35, 1842-1858 (2012).

21. Catapano, A.: Stiffness and strength optimisation of the anisotropy distribution for laminated structures. $\mathrm{PhD}$ thesis, University Paris 6, http://tel.archives-ouvertes.fr/tel00952372 (2013).

22. Vincenti, A. and Desmorat, B.: Optimal orthotropy for minimum elastic energy by the polar method. Journal of Elasticity, 102, 55-78 (2010).

23. Julien, C.: Conception Optimale de l'Anisotropie dans les Structures Stratifiees à Rigiditè Variable par la Méthode Polaire-Génétique. PhD thesis, University Paris 6 (2010).

24. Vannucci, P. and Verchery, G.: A special class of uncoupled and quasi-homogeneous laminates. Composite Science and Technology, 61, 1465-1473 (2001).

25. Vannucci, P.: Designing the elastic properties of laminates as an optimisation problem: a unified approach based on polar tensor invariants. Structural and Multidisciplinary Optimisation, 31, 378-387 (2006).

26. Vannucci, P. and Vincenti, A.: The design of laminates with given thermal/hygral expansion coefficients: a general approach based upon the polar-genetic method. Composite Structures, 79, 454-466 (2007).

27. Montemurro, M., Vincenti, A. and Vannucci, P.: Design of elastic properties of laminates with minimum number of plies. Mechanics of Composite Materials, 48, 369-390 (2012).

28. Montemurro, M., Vincenti, A. and Vannucci, P.: A two-level procedure for the global optimum design of composite modular structures - application to the design of an aircraft wing. part 1: theoretical formulation. Journal of Optimization Theory and Applications, 155, 1-23 (2012). 
29. Vincenti, A.: Conception et optimisation de composites par la méthode polaire et algorithmes génétiques. PhD thesis, University of Burgundy (2002).

30. Vincenti, A., Ahmadian, M. R. and Vannucci, P.: Bianca: a genetic algorithm to solve hard combinatorial optimisation problems in engineering. Journal of Global Optimization, 48, 399-421 (2010).

31. Montemurro, M., Vincenti, A. and Vannucci, P.: A two-level procedure for the global optimum design of composite modular structures - application to the design of an aircraft wing. part 2: numerical aspects and examples. Journal of Optimization Theory and Applications, 155, 24-53 (2012). 